\title{
Eliciting Preferences for HIV Prevention Technologies: A Systematic Review
}

\author{
S. Wilson Beckham ${ }^{1} \cdot$ Norah L. Crossnohere ${ }^{1,2}\left(\right.$ Margaret Gross $^{3} \cdot$ John F. P. Bridges ${ }^{1,2}$
}

Accepted: 18 November 2020 / Published online: 15 December 2020

(c) The Author(s) 2020

\begin{abstract}
Background Many human immunodeficiency virus (HIV) prevention technologies (pre-exposure prophylaxis, microbicides, vaccines) are available or in development. Preference elicitation methods provide insight into client preferences that may be used to optimize products and services. Given increased utilization of such methods in HIV prevention, this article identifies and reviews these methods and synthesizes their application to HIV prevention technologies.

Methods In May 2020, we systematically searched peer-reviewed literature in PubMed, CINAHL, and Web of Science for studies employing quantitative preference elicitation methods to measure preferences for HIV prevention technologies among populations of any age, sex, or location. Quality assessment used an existing checklist (PREFS) and a novel adaptation of the Newcastle-Ottawa Scale (PROSPERO \#CRD42018087027).

Results We screened 5022 titles and abstracts, reviewed 318 full texts, and included 84 studies. Common methods employed were discrete-choice experiment (33\%), conjoint analysis (25\%), and willingness-to-participate/try/accept (21\%). Studies were conducted in 25 countries and had a mean of 768 participants (range $=26-7176$ ), two-thirds of them male. Common HIV prevention technologies included pre-exposure prophylaxis (23\%), voluntary testing and counseling (19\%), HIV self-testing (17\%), vaccines (15\%), and topical microbicides (9\%). Most attributes focused on product design (side effects, frequency), service design (provider type, location), acceptability or willingness to accept/pay; results are summarized in these categories, by prevention type. Mean quality-adapted Newcastle-Ottawa Scale score was 4.5/8 (standard deviation=2.1) and mean PREFS scores was 3.47/5 (standard deviation=0.81). Conclusions This review synthesizes extant literature on quantitative measurement of preferences for HIV prevention technologies. This can enable practitioners to improve prevention products and interventions, and ultimately reduce HIV incidence.
\end{abstract}

\section{Introduction}

In the first decades of the human immunodeficiency virus (HIV) epidemic, biomedical options for prevention were scant, with condoms the only option for prevention of sexual transmission [1]. Other prevention options included postexposure prophylaxis (PEP) for occupational exposure, promotion of testing, nevirapine for prevention of motherto-child transmission (PMTCT), syringe exchange, and medication-assisted treatment (MAT) to reduce the risks of

Norah L. Crossnohere

norah.crossnohere@ osumc.edu

1 Department of Health, Behavior and Society, Johns Hopkins Bloomberg School of Public Health, 624 N Broadway, Baltimore, MD 21205, USA

2 Department of Biomedical Informatics, The Ohio State University College of Medicine, Columbus, $\mathrm{OH}$, USA

3 College of Veterinary Medicine, North Carolina State University, Raleigh, NC, USA injection drug use and thus HIV risk [1,2]. This menu of choices has expanded recently to include voluntary medical male circumcision (VMMC); pre-exposure prophylaxis (PrEP) pills, now in two formulations; and treatment as prevention [3-6]. Blood-based rapid HIV tests and oral fluidbased rapid HIV self-tests for use at home seek to expand the number of people aware of their HIV status, and thus constitute a key first step on the HIV prevention and treatment cascades [7,8].

Several HIV prevention technologies are in development or testing. These include vaccines, topical microbicides, and multiple formulations or modalities of PrEP, such as injectables, vaginal rings, implants, and diaphragms $[9,10]$. A trial of bimonthly long-acting injectable cabotegravir was recently stopped early, owing to its proven effectiveness compared to oral daily PrEP pills [11]. The future of HIV 


\section{Key Points for Decision Makers}

As preference elicitation methods are increasingly used in health services research, including HIV prevention, quality standards and criteria are needed to ensure use of accurate terminologies and appropriate methods and analytic tools.

To grow as a field, we need to build on each other's work, including learning from already existing literature, synthesizing data instead of generating data for data's sake, utilizing and implementing findings, and re-using existing instruments as appropriate rather then creating new tools.

Trends in use of particular preference elicitation methods, focus on particular outcomes and health topics, e.g., HIV prevention technologies, and populations/geographies, should be critically examined for their trends in popularity and funding sources.

prevention may look more like the contraceptive marketplace, with a menu of options that individuals can choose from according to their risks, needs, and preferences [12].

Quantitative preference elicitation methods measure and provide insight into client preferences. These methods may then be used to optimize product design while still in development (i.e., gel consistency; oral swab vs finger prick), or inform implementation strategies or service design (dispensing location, cost, healthcare provider characteristics) for products already or soon to be on the market. These methods have been increasingly applied to health issues, including HIV/acquired immunodeficiency syndrome prevention and treatment [13] and health system priorities [14]. With such patient-centered data, products and services can potentially be adapted to better suit target audiences. For example, the HIV prevention needs of a seasonal female sex worker in Nairobi and of a gay man in Atlanta are disparate, even if both opt for PrEP. Human immunodeficiency virus prevention options that better meet different audiences' needs may potentially improve uptake, adherence, and persistence (long-term use), ultimately reducing HIV incidence.

Given this growing landscape of HIV prevention technologies along with the increasing application of quantitative preference elicitation methods to HIV-related topics, this review summarizes the current literature and identifies methodological norms in this rich and growing literature. We also advance the critical appraisal of reporting on stated-preference experiments by administering both the PREFS checklist (purpose of study, respondent sampling, explanation of assessment methods, inndings, significance testing) [15] as well as a customized Newcastle-Ottawa tool [16].

\section{Methods}

\subsection{Inclusion and Exclusion Criteria}

\subsubsection{Populations}

We included populations of any age or sex, or other stakeholders concerned with preventing HIV transmission. Preferences of people living with HIV were included if the target intervention was prevention, e.g., PMTCT, but otherwise excluded.

\subsubsection{Interventions}

We considered HIV prevention technologies either currently available or under development: PrEP in any form (tablet, ring, injectable); topical microbicides; cervical barriers and intravaginal devices; clean injecting equipment; MAT; voluntary testing and counseling; HIV self-testing; VMMC; PMTCT; PEP; and external (male) and internal (female) condoms. We excluded prevention technologies used for purposes other than prevention of HIV (condoms only as contraception), unless preferences were elicited for the product in combination with its HIV prevention use. We excluded preference elicitation concerning strictly behavioral interventions without the aid of a biomedical, biotechnological, or pharmaceutical intervention.

While HIV testing is not itself a prevention intervention, we included it in this review for two reasons. One, there are technological advances in HIV testing, particularly newer rapid tests and blood- and saliva-based self-test kits, which make them a popular subject of preference elicitation methods. Further, HIV testing is considered an essential part of the HIV prevention and treatment cascades, as an initial step in primary and secondary prevention [7, 8]. While HIV tests in and of themselves do not biomedically prevent HIV, as VMMC and PrEP do, testing is nevertheless an important part of the HIV prevention technology landscape.

\subsubsection{Types of Studies}

We included papers that used quantitative preference elicitation in their study design. We excluded papers that describe preferences through solely qualitative methods. The definition of quantitative preference elicitation methods (i.e., stated-preferences methods) followed Soekhai et al. [17], and included discrete-choice experiments (DCEs); conjoint analysis (CA); best-worst scaling (BWS) cases 1, 2, and 3; 
willingness to pay (WTP); willingness to accept, try, or participate (WTA); contingent valuation (CV); Q-methodology; control preferences scale; self-explicated methods; analytic hierarchy processes; standard gamble; time trade-off; person trade-off; test trade-off; starting known efficacy; constant sum scaling; repertory grid method; threshold technique; swing weighting; visual analog scale; point allocation; and outcome prioritization methods. Studies that used only Likert scales, rating, or ranking were excluded. We classified study types by how they were named by the studies' authors, with some exceptions. First, if the study did not use any of the above terms (e.g., called it simply a "choice experiment"), the reviewers (SWB, JFPB, NC) discussed the description of the elicitation method and determined which it fit most closely. Second, the authors of a small number of studies labeled experiments CA, but the description of the methods indicated they used DCE methods. We reclassified these as DCEs [18].

\subsubsection{Outcomes}

The co-primary outcomes were (1) the prevention technology type investigated (e.g., PrEP); and (2) the preference elicitation method applied (e.g., DCE).

\subsection{Search Strategy}

With the assistance of an informaticist, we developed the search strategy and conducted searches in November 2017, with updates in March 2019 and May 2020. We developed search terms for PubMed, then adapted as relevant for Web of Science and CINAHL databases (see Appendix). We used a combination of controlled vocabulary and keywords for (1) human immunodeficiency virus, (2) individual prevention technologies, and (3) preference elicitation methods, with no restrictions on language, publication date, or geography. The PROSPERO registration is \#CRD42018087027. We hand searched references from selected studies and review articles to identify any additional relevant studies.

\subsection{Screening and Review}

Data screening and review were conducted in Covidence (Veritas Health Innovation, Melbourne, VIC, Australia, http://www.covidence.org). We conducted dual screening of titles and abstracts (SWB, NLC); one or two affirmative votes resulted in a full-text review. Two readers (SWB, NLC) independently reviewed full texts to confirm inclusion. We excluded records that were only abstracts or the full text was otherwise unavailable. The two reviewers discussed disagreements to reach a consensus, with a third reviewer (JFPB) if necessary.

\subsection{Data Abstraction}

SWB abstracted the following information, with unclear information reviewed by another author (NLC or JFPB): author, year of publication, title, location, population, sample size, first author affiliation, funding source(s), quantitative preference elicitation method, HIV prevention technology, number of attributes, strategies for attribute identification, data collection tool, statistical analysis approaches, and summary of main findings.

\subsection{Quality Assessment}

NLC rated study quality using two quality assessment tools. (1) The PREFS checklist, an existing and widely used tool [19-21] to assess reporting on factors that may influence the validity of patient preference studies: purpose of study, respondent sampling, explanation of assessment methods, findings, significance testing, with one point each (score 0-5) [22]. (2) The Newcastle-Ottawa Scale (NOS): crosssectional studies [16], here adapted to reflect quality considerations for patient preference studies (see Appendix). The NOS contains seven items (representativeness, sample size, non-respondents, exposure assessment, comparability, outcome assessment, and statistical tests). The NOS employs a rating system that uses both letters grades $(\mathrm{A}, \mathrm{B}, \mathrm{C})$ and stars $(*)$, where more stars indicate higher quality. For this review, we dropped the "outcome assessment" category because outcomes are always collected via self-report in patient preference studies; this is a feature of preference work rather than a flaw. Our adapted version replaced "exposure assessment" with "validity of preference elicitation," (validated measurement tool used, or a clearly explained and rigorous instrument development process). We also replaced "comparability" with "heterogeneity" (whether preference differences had been explored across sub-groups or segments of respondents). The highest score was eight stars (following the original NOS, "validity" and "heterogeneity" could earn up to two stars each).

We also conducted an exploratory analysis to describe variations in study quality across publication date and by method of preference elicitation. We created binary PREFS and NOS scores based on a median split of each variable, studies with PREFS scores of 1,2, and 3 were described as "low-quality PREFS" and studies with PREFS scores of 4 and 5 were recoded as "high-quality PREFS." Studies with NOS scores lower than 6 were described as "low-quality NOS" and those with NOS scores of 6 or greater were described "high-quality NOS." T-tests of binary PREFS and NOS variables and years since publication explored differences in study quality over time. Chi-squared tests explored differences in distribution of high- and low-quality NOS and PREFS scores for each of the methods (BWS, DCE, CA, CV, 
WTP, WTA). All analyses were conducted using Stata Version 16 (StataCorp. 2019. Stata Statistical Software: Release 16. College Station, TX: StataCorp LLC.).

\section{Results}

Searches yielded 6944 records, and 5022 titles and abstracts to screen after we removed duplicates. After a full text review of 318 articles, 84 manuscripts were eligible and included. The most common reason for exclusion at the fulltext review was that the methods did not meet the definition of quantitative preference elicitation $(\mathrm{k}=162)$. See Fig. 1 for the PRISMA (Preferred Reporting Items for Systematic Reviews and Meta-Analyses) diagram. Study characteristics are found in Table 1. Note: $k$ refers to the number of included studies, and $n$ refers to the number of participants within the studies.

\subsection{Study Descriptions and Methodologies}

\subsubsection{Preference Elicitation Methods}

The most common preference elicitation methods used were DCE $(28,33 \%)$, CA $(21,25 \%)$, WTA $(18,21 \%)$, and WTP [including CV] $(15,18 \%)$. Only two used BWS [23, 24], and no other preference elicitation methods were found. The oldest article was from 1998; it used WTA and WTP direct elicitations to examine women's preferences for vaginal antimicrobial (sexually transmitted infection [STI]/HIV) contraceptives [25]. There was a clear increase in the number of publications in the past decade, with $83 \%$ of the included studies published in 2010 or later; the median publication year was 2016. Of the 15 studies published in 2019 and 2020, 12 were DCE (see Fig. 2a, b).

\subsubsection{Attribute Identification}

The range in the number of attributes was 1-11 (mean 4.14, median 5; excluding three studies where the number of attributes was unclear). Excluding studies that had only one attribute, i.e., WTP $(26,31 \%)$, and studies where the number of attributes was unclear (3), the mean number of attributes was 5.98 and median was 6. A Task Force on Good Research Practices for Preference Elicitation Experiments recommended a variety of strategies (literature review, qualitative formative work, consultation of expert opinion, pre-testing [e.g., face-to-face qualitative interviews or cognitive interviewing on the instrument], and pilot testing [administration of the instrument to a subset of the final sample]) to identify attributes and levels for preference elicitation experiments [26]. Many of the studies $(36,43 \%)$ did not report any of these (58\% of those were investigating willingness). Only $42 \%$ clearly reported conducting literature reviews, $45 \%$ formative (usually qualitative) research, $24 \%$ expert consultations, $8 \%$ pre-testing, and $20 \%$ pilot testing (see Fig. 3 ).
Fig. 1 PRISMA (Preferred Reporting Items for Systematic Reviews and Meta-Analyses) diagram. HIV human immunodeficiency virus, quant. pref. quantitative preference

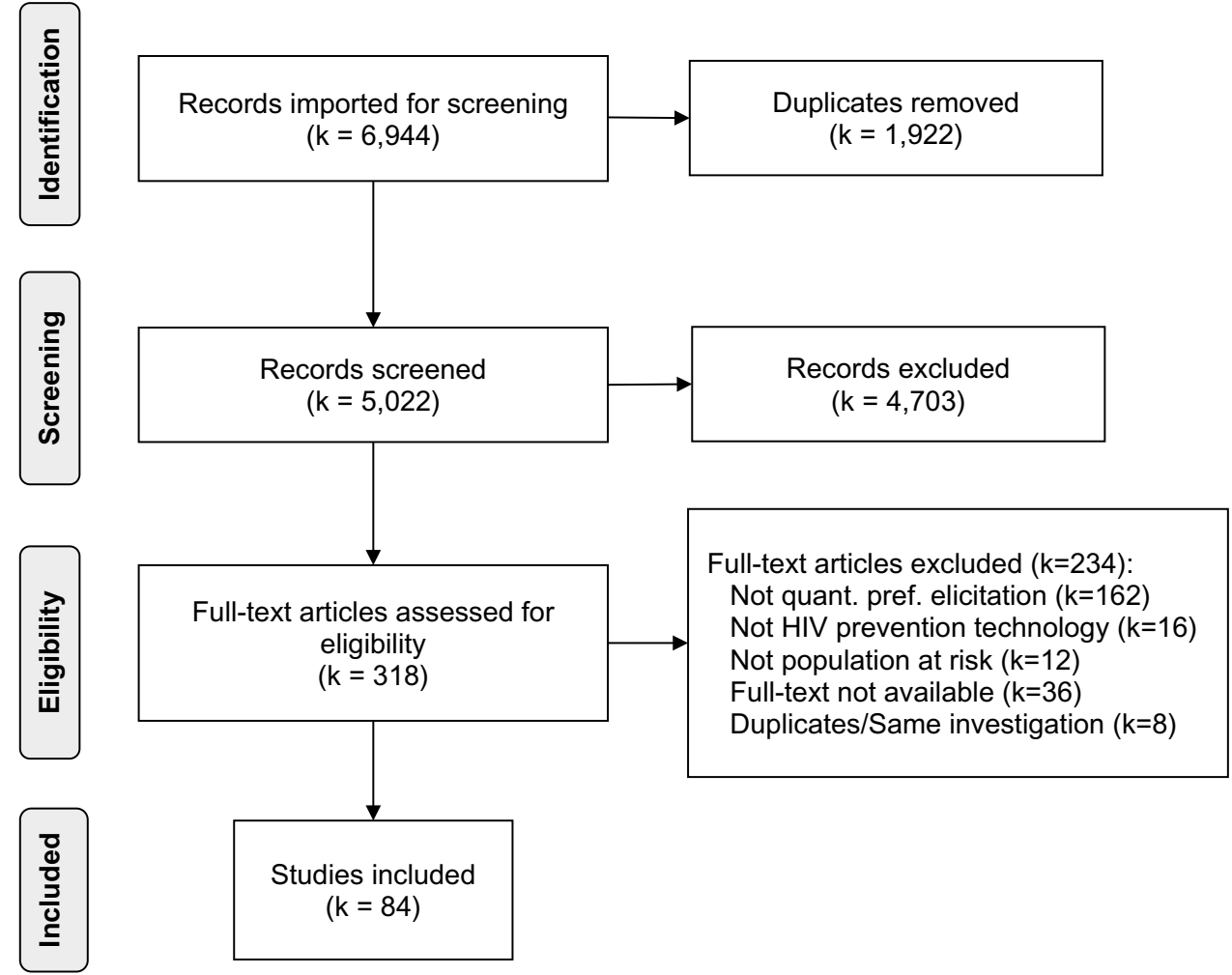




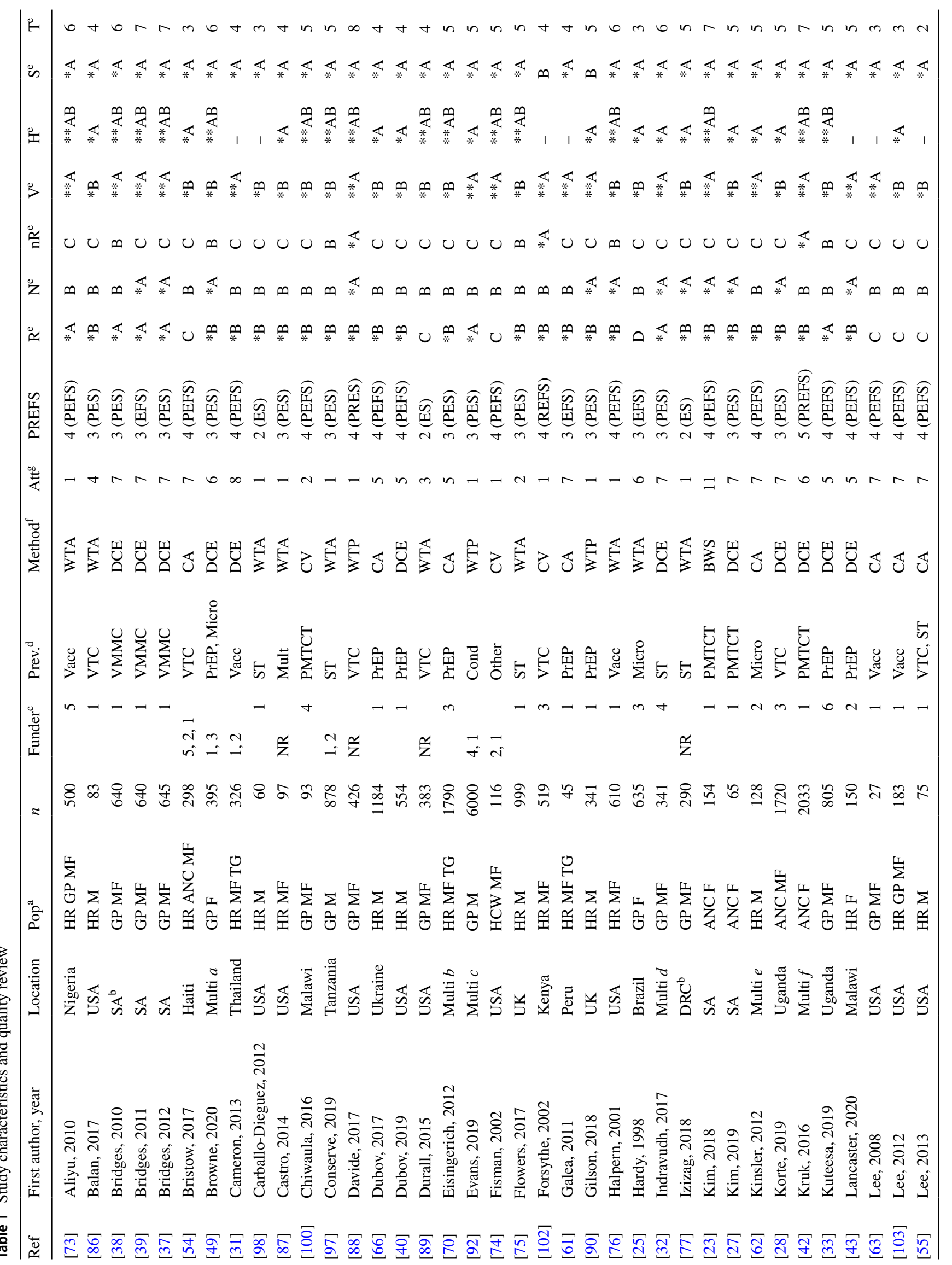




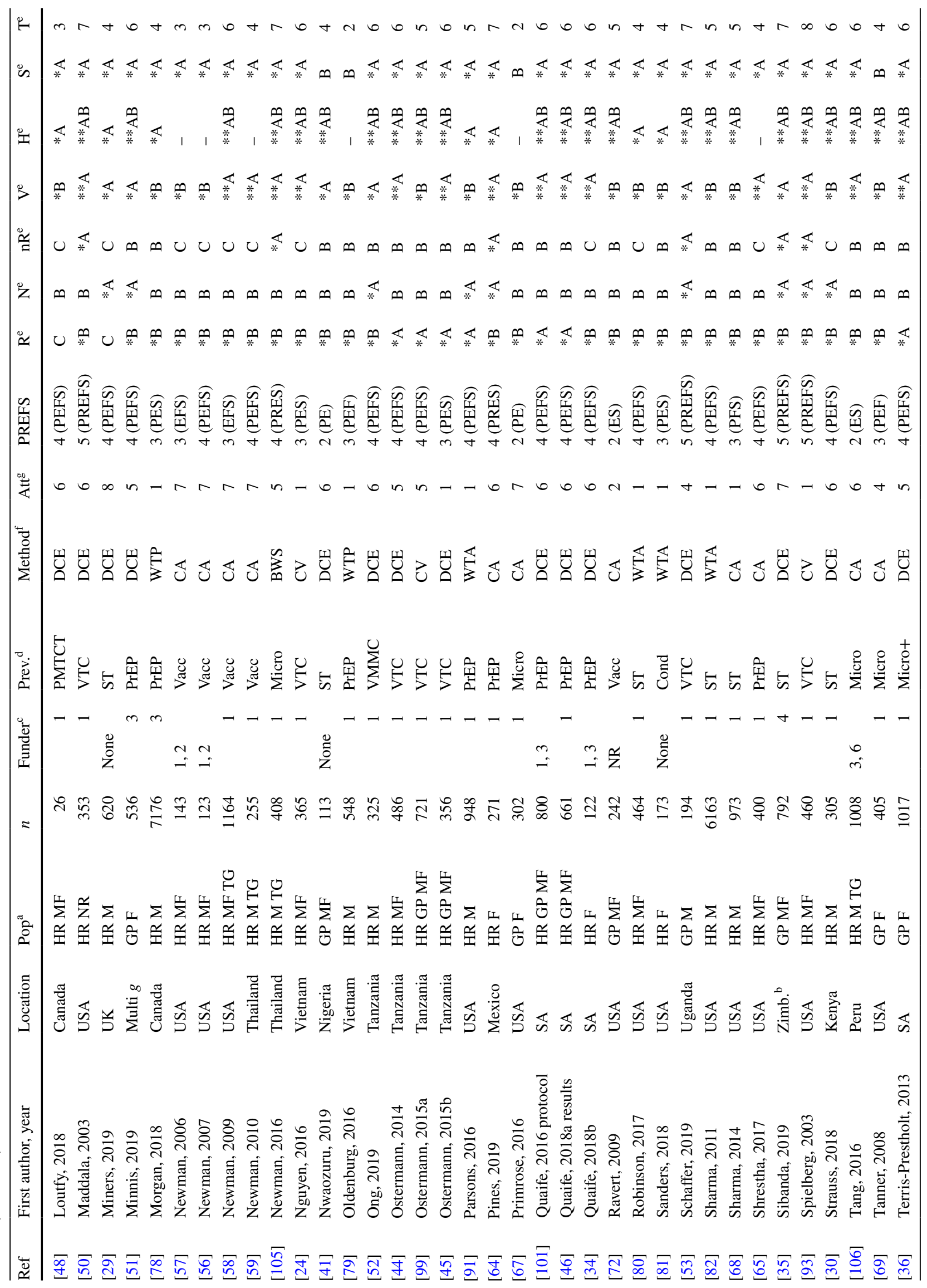




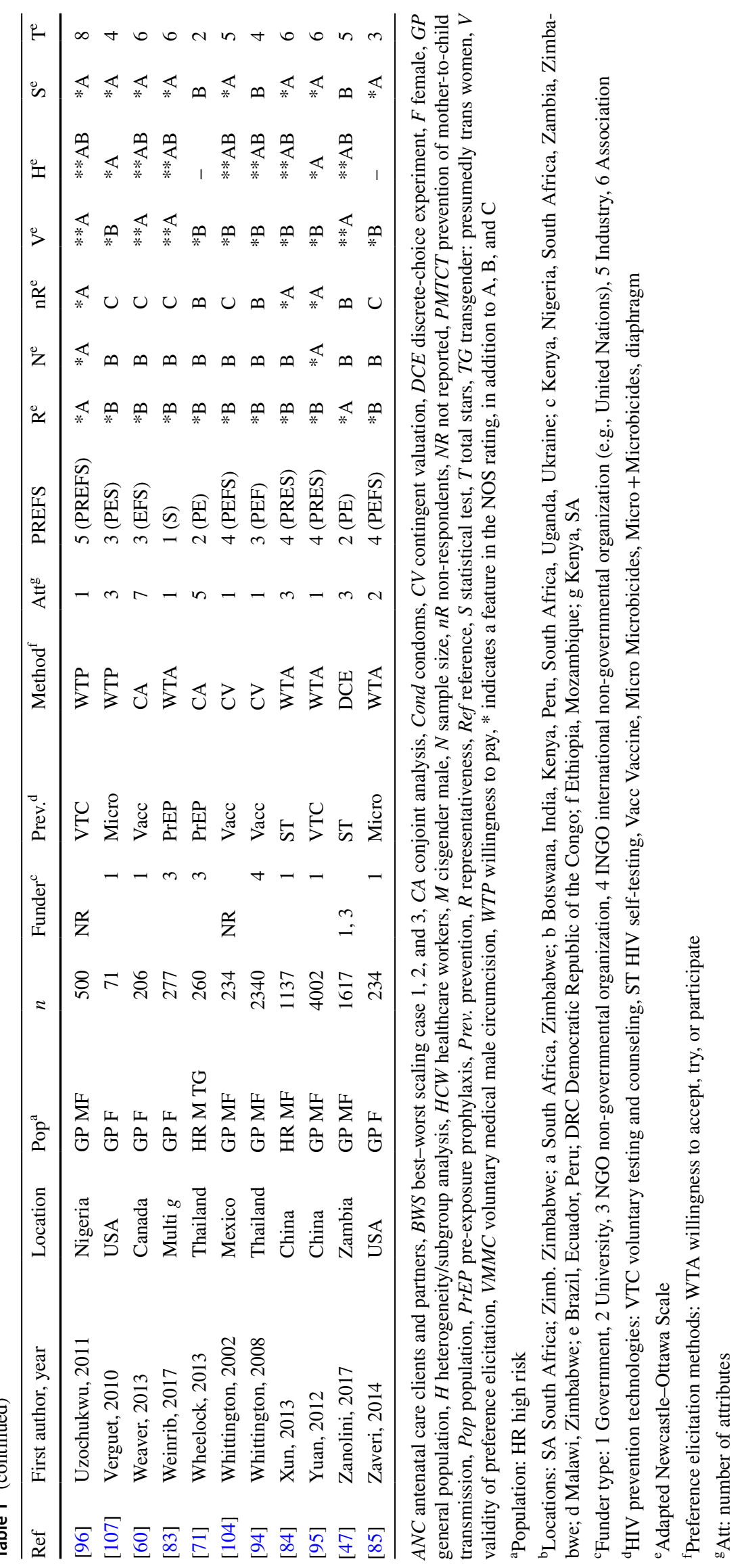




\subsubsection{Data Analysis}

The included studies reported a variety of methodological approaches and statistical analysis methods, and because the various elicitation methods require different analyses, we consider DCE, BWS, CA, and willingness studies separately in this section. Note that appropriateness of statistical tests and measurement of preference heterogeneity are included in the Quality Review section and Table 1.

3.1.3.1 DCE Of the 28 studies that employed DCE, several base statistical models were reported, including conditional logit $(4,14 \%)$ [27-30], multinomial logit $(5,18 \%)$ [31-36], ordinary least-squares regression $(2,7 \%)[37,38]$, and logistic regression (1) [39]. Three (11\%) reported relative impor- tance scores [32, 40, 41]. Several studies also conducted mixed-logit $(8,29 \%)$ [33, 34, 42-47], nested-logit $(3,11 \%)$ $[35,36,48]$, or random parameters logit $(7,25 \%)[32,35$, 49-53] (some studies reported more than one model). Most of the DCE studies $(24 / 28,86 \%)$ further measured preference heterogeneity using stratification/subgroup analyses and/or interaction terms $(17,61 \%)$, or latent class analysis $(6,21 \%)[29,33,40,46,51,52]$. One study used a blocked design with two different versions of the DCE for the two a priori sub-groups (past HIV testers vs non-testers) [47.]

3.1.3.2 BWS The two BWS studies used different types of analyses. Kim et al. reported relative and mean BWS scores, paired t-tests, and linear regression [23]. Newman et al. used
Fig. 2 Publications by year and preference elicitation method (a), and publications by year and human immunodeficiency virus prevention method (b). $C V$ contingent valuation, $P M T C T$ prevention of mother-to-child transmission, $\mathrm{PreP}$ pre-exposure prophylaxis, $V M M C$ voluntary medical male circumcision

\section{a Publications by Year and Preference Elicitation Method}

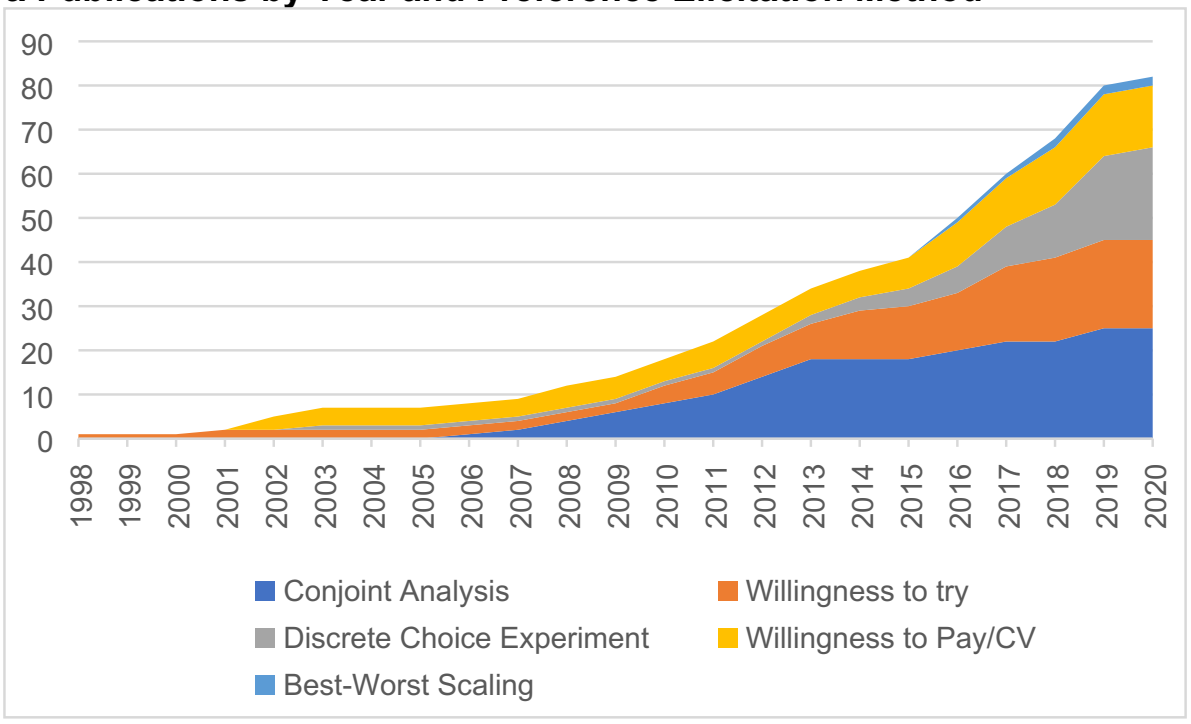

\section{b Publications by Year and HIV Prevention Method}

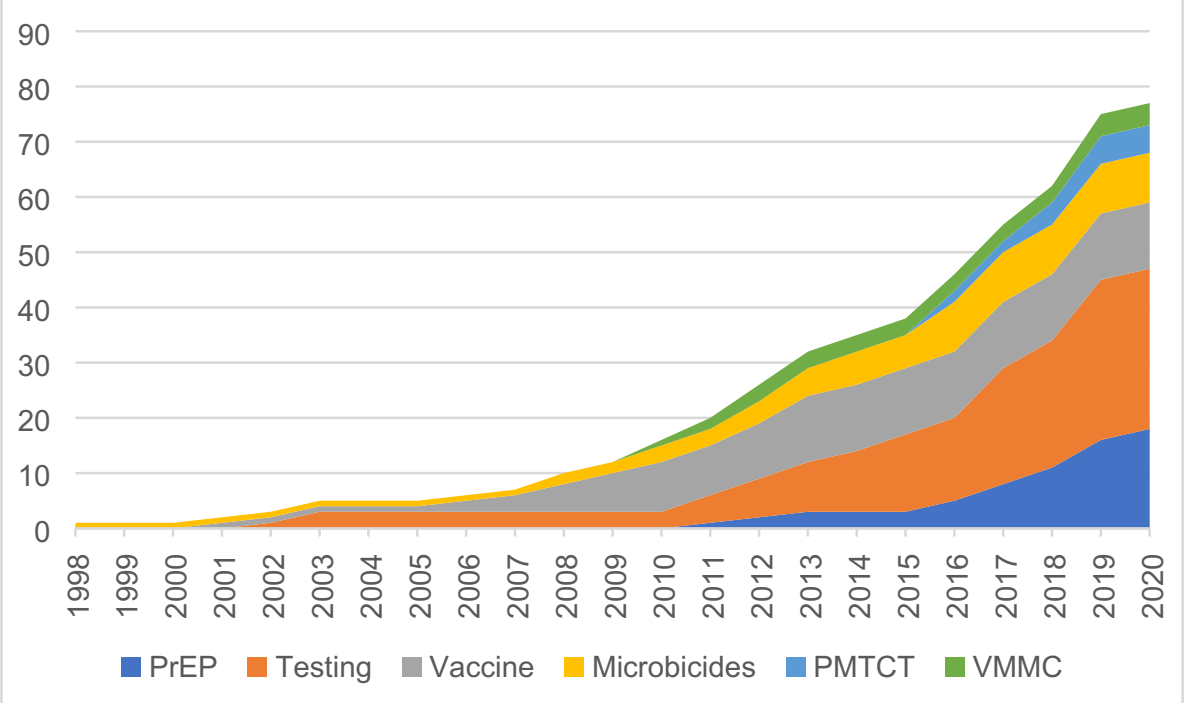




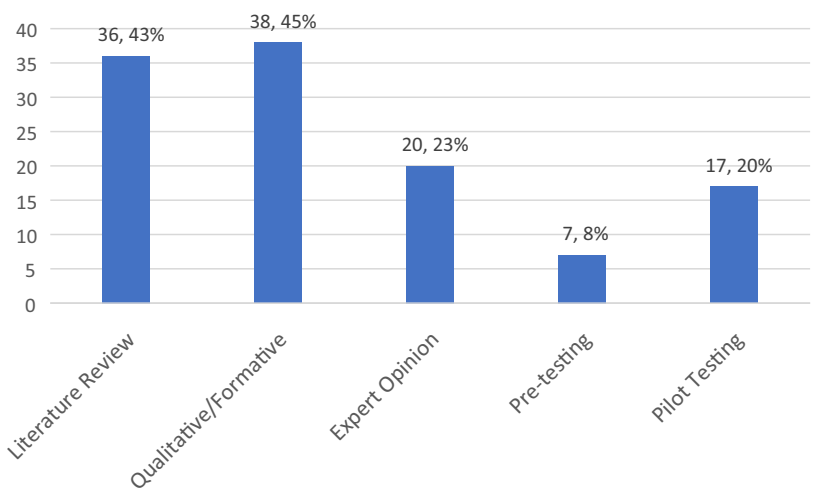

Fig. 3 Attribute identification strategies employed, recommended by ISPOR [26]

multinomial logit and rank-exploded logit. Both also used stratification to measure preference heterogeneity.

3.1.3.3 CA Of the 21 CA studies, analysis methods included individual-specific, mean, and/or relative impact scores $(7,33 \%)$ [54-60]; mean ratings $(5,24 \%)$ [61-65], relative importance scores or interest values $(4,19 \%)$ [64-67]; modified Borda count method (1) [68]; part-worth utilities (4, 19\%) [64-66, 69]; hierarchical Bayes estimation (2, 10\%) $[70,71]$, and linear regression (1) [72]. To measure preference heterogeneity, two CA studies used subgroup analyses or stratification $[69,70]$; and one used latent class analysis [66].

3.1.3.4 Willingness/CV Studies There were 33 studies that measured WTP/WTA, including eight that used CV approaches to WTP. A variety of statistical approaches were used in these studies, most commonly logistic regression $(13,39 \%)$ [73-85] and Chi squared tests (including Pearson's and Fisher's exact) $(12,36 \%)[24,25,73,75,80,83$, $86-91]$. T-tests $(5,15 \%)$ and analysis of variance $(4,12 \%)$ [24, 86, 88, 91] were also employed, as well as types of regression (linear [92, 93], count [94], log binomial [95], ordinary least squares [96], and logit link [97]). Three studies used Mann-Whitney U tests $(9 \%)$ [81, 93, 98]. One study each reported using the area under a receiver operating characteristic curve [76], maximum likelihood double-bounded, dichotomous choice CV model [99], Wilcoxon signed-rank [100], Kruskal-Wallis [24], and Bernoulli response [97]. Two CV studies [94, 99] and one WTA [84] used stratification to measure preference heterogeneity.

\subsubsection{HIV Prevention Technologies}

The most common HIV prevention technologies were PrEP $(19,23 \%)$, voluntary testing and counseling $(16,19 \%)$, HIV self-testing $(14,17 \%)$, vaccines $(13,15 \%)$, and topical microbicides $(8,9 \%)$. Less commonly investigated were PMTCT (5, 6\%), VMMC (4, 5\%), and condoms (2, 2\%). Two studies were classified as "other": one was a needlestick prevention device [74], and one had multiple prevention technologies (external and internal condoms, topical microbicides, VMMC, PrEP, and vaccine) [87]. The highest number of publications in one year was in $2019(13,15 \%)$, with five of those on PrEP and six on HIV testing. PrEP, PMTCT, and VMMC studies were all published in the past decade, and no vaccine preference studies were published after 2013. See below for a synthesis of the included attributes and main findings.

\subsubsection{Populations}

Included studies were conducted in 25 countries on five continents. Most were conducted in sub-Saharan Africa (SSA) $(45,54 \%)$ and North America $(30,36 \%)$. There were ten $(12 \%)$ in Latin America and the Caribbean, ten (12\%) in Asia (six of those in Thailand), and only six (7\%) in Europe (UK and Ukraine only). The USA (27, 32\%) and South Africa $(14,17 \%)$ had the greatest number of studies.

The total number of participants in all studies was $n=61,446$, mean $n=768$, median $n=374$, and range 26-7176 (not double counting the same participants reported in more than one publication [37-39, 44, 45, 101]). The total number of participants in the DCE/BWS studies was $n=14,866$, mean $n=495.5$, median $\mathrm{n}=347$, and range 26-2033. The total total number of participants in the CA studies was $n=9482$, mean $n=451.5$, median $n=260$, and range 27-1790. The total total number of participants in the WTA/WTP/CV studies was $n=34,241$, mean $n=1037.6$, median $n=460$, and range 60-7176.

Of the participants, $68 \%$ were male $(n=41,562), 31 \%$ were female $(n=19,017$ cisgender female, and $n=261$ transgender female), and $1 \%$ of participants did not report sex/gender $(n=603)$. About half of the studies $(45,54 \%)$ included key populations at high risk for HIV (e.g., sex workers, men who have sex with men [MSM], clients of STI clinics); six (7\%) included antenatal care clients, and two included both high-risk and general populations. The rest $(29,35 \%)$ were conducted among general populations, though $15(52 \%)$ of those studies were in high HIV prevalence countries where segments of the general population are at elevated risk (e.g., young women in Kenya). One study was among healthcare workers (WTP for a needle-stick prevention device) [74], and one among informal caregivers of women living with HIV (WTP for PMTCT-related caregiving, and to be paid for caregiving) [100]. 


\subsubsection{Survey Administration}

Thirty-two studies (38\%) were paper surveys, 27 (32\%) computer-assisted self-interview/personal interviewing software or tablets, $11(13 \%)$ online, and one telephone interview. The remaining studies $(13,15 \%)$ did not report this information. The majority $(54,64 \%)$ had facilitators present to either conduct the surveys or assist with computer-assisted self-interview/personal interviewing software, 20 (23\%) did not, and $11(13 \%)$ did not clearly state this information.

\subsubsection{Author Affiliation and Funding}

The majority of the studies were conducted by investigators at universities $(71,84 \%)$, with others conducted by nongovernmental organizations and institutes $(8,9 \%)$, hospitals $(3,4 \%)$, industry (1), or government health programs (1). Funders of included studies were largely government research institutions [e.g., National Institutes of Health in the USA] $(48,57 \%)$ and government development agencies [e.g., Department for International Development in the UK] $(13,15 \%)$. Private foundations [e.g., Bill and Melinda Gates Foundation] $(10,12 \%)$, research institutes $(5,6 \%)$, intergovernmental organizations [e.g., United Nations] (7, 8\%), and non-governmental organizations $(4,5 \%)$ comprised the remaining funders. Ten studies (12\%) did not report funding.

\subsection{Synthesis of Included Attributes, Levels, and Main Findings}

Presented below is a qualitative synthesis of the attributes as well as the key findings for each HIV prevention modality. We grouped the attributes into three main categories: product design, service design (or program implementation), and willingness/acceptability. See Table 2 for a list of attributes and levels by HIV prevention technology. A handful of studies fell outside these categorizations; see below.

\subsubsection{Pre-Exposure Prophylaxis}

Nineteen studies examined PrEP [33, 34, 36, 40, 43, 46, 49, 51, 61, 64-66, 70, 71, 78, 79, 83, 90, 91, 101].

3.2.1.1 Product Design The most common PrEP product design attributes were frequency of dosing (daily, monthly) and product formulation, which necessarily overlap (pills: daily; injections: monthly). Some studies found that dosing frequency was unimportant compared to other attributes [43, 61, 79], while other studies showed preferences for daily pills [33, 40, 65, 71], for monthly or bimonthly injections or implants [51, 70, 91], or for on-demand dosing (with sexual encounters) of rectal gels [64, 79]. Formulation (pills, injections, rings, gel) was found to be variously as important as efficacy [29], even more important than efficacy [64], or secondary to efficacy [91]. In studies that considered the vaginal ring, the ring rated poorly compared with other formulations, and was sometimes the least preferred [33, 34, 51, 64, 83]. Other product attributes included side effects, efficacy, additional protection from pregnancy or STI (pregnancy prevention was valued; STI prevention was valued among female sex workers [FSW]) [46, 49, 83]; and changes to menstruation [51].

Subgroup analyses showed heterogeneity in formulation preferences. Some MSM in the USA and Ukraine preferred injections, others on-demand pills, and others were uninterested in PrEP [40, 66]. In Uganda, while pills were overall preferred, women who used alcohol, experienced intimate partner violence in the past year, and did not make household decisions preferred implants and injections over pills [33]. Both general population women and FSW in South Africa preferred injections, while men cared little about formulation [46].

3.2.1.2 Service Design Pre-exposure prophylaxis service design attributes most found were cost (US\$0-250; out of pocket; insurance coverage) and location of dispensing site (HIV clinic, drop-in center, mobile clinic). Cost was less important than formulation to some [64], but it was the most important attribute in other studies [61, 65]. In subgroup analyses of MSM in the USA, cost was important in every group, while the importance of other attributes varied [40]. For dispensing sites, FSW in Malawi most valued family planning clinics or drop-in centers [43], FSW near the US-Mexico border preferred healthcare clinics vs nongovernmental organizations [64], MSM in Thailand and the USA preferred pharmacies [40,71], and people who used drugs in the USA preferred HIV clinics over drug treatment clinics [65]. Dispensing site was the most important attribute in African countries in a multinational study; dispensing at HIV treatment clinics was highly devalued; the authors hypothesize this was related to HIV stigma [70]. Frequency of HIV testing while using PrEP was examined in three studies; semi-annually was preferred over more frequent testing in all three studies $[65,70,71]$.

3.2.1.3 Willingness Two studies measured WTP for PrEP. Both were conducted in high-income countries (Canada, Scotland). Scottish men were willing to pay $£ 50$ monthly for PrEP, but a high proportion of Canadian respondents would only use it if covered by insurance $[78,90]$.

3.2.1.4 Risk Compensation One study measured potential risk compensation during PrEP use among FSW in South Africa (they and/or their clients may be less willing to use condoms or accept/pay more for condomless sex). The authors found that PrEP use may negatively impact willing- 
Table 2 Attributes and levels used by included studies in prevention technology

\begin{tabular}{|c|c|c|c|c|}
\hline Attribute & Level examples & $k$ & Attribute & Level examples \\
\hline \multicolumn{5}{|c|}{ Pre-exposure prophylaxis $[33,34,40,43,46,49,51,61,64-66,70,71,78,79,83,90,91,101]$} \\
\hline \multicolumn{5}{|c|}{ Product design } \\
\hline Frequency (dosing, refill) & $\begin{array}{l}\text { Daily, on demand, monthly, } \\
\text { bimonthly }\end{array}$ & 9 & Efficacy/dffectiveness & $30 \%, 50 \%, 80 \%, 99 \%$ \\
\hline Formulation/modality & $\begin{array}{l}\text { Pill, injection, vaginal ring, gel, } \\
\text { implant }\end{array}$ & 7 & STI prevention & Yes/no \\
\hline Side effects & $\begin{array}{l}\text { Nausea, dizziness; mild, moder- } \\
\text { ate }\end{array}$ & 4 & Menstruation changes & Light/regular, heavy/irregular \\
\hline Pregnancy prevention & Yes/no & 3 & & \\
\hline \multicolumn{5}{|l|}{ Service design } \\
\hline Cost & $\begin{array}{l}\text { US\$0-50; out-of-pocket, insur- } \\
\text { ance }\end{array}$ & 7 & Adherence support & Peer, online, phone, face-to-face \\
\hline Location & $\begin{array}{l}\text { HIV clinic, drop-in-center, } \\
\text { mobile }\end{array}$ & 7 & Duration of use & 1,10 years \\
\hline Frequency of HIV testing & Monthly, semiannually & 3 & Provider type/characteristics & $\begin{array}{l}\text { Sex, age; doctor, nurse, lay } \\
\text { person }\end{array}$ \\
\hline Time spent obtaining & $2,4 \mathrm{~h}$ & 2 & Other screening on site & $\begin{array}{l}\text { Cervical cancer, pregnancy } \\
\text { prevention }\end{array}$ \\
\hline Pre-prescription evaluation & $\begin{array}{l}\text { Least, moderate, most demand- } \\
\text { ing }\end{array}$ & 2 & & \\
\hline
\end{tabular}

Traditional HIV voluntary testing and counseling [24, 28, 44, 45, 50, 53-55, 86, 88, 93, 95, 96, 99, 102]

\section{Product design}

Fluid sampling method

Accuracy (syphilis testing)

\section{Service design}

Cost

Location

Pre/post-test counseling type

Time (to result/entire visit)

ART availability
Privacy/confidentiality
Provider type/characteristics
Schedules/opening hours

Oral swab, finger prick, venous

Almost always, less

US\$0, US\$1, US\$4, US\$30

Clinic near home, at home

Telephone, leaflet, in person

20 min, 1 week

On site, immediately, delayed

Anonymous, confidential, (not) linked

Dentist, lay worker; age

Weekends, weekdays, evenings 2
8 Number of blood draws

1 STI testing

6 Distance to services

5 Other screening on site

4 Monetary incentive

4 Distribution/delivery strategies

3 Test type

3 How obtain results

2 Partner/family support

2

HIV self-testing [29, 30, 32, 35, 41, 47, 68, 75, 77, 80, 82, 84, 89, 97, 98]

Product design

Fluid sampling method

Accuracy

Oral, finger prick, venous, blood 4 Infection window period spot

Service design

Location

Cost/user fee

Post-test counseling type

Provider type/characteristics

$95 \%, 99 \%$

1 Other testing in same test

Facility, mobile clinic, home

8 Time (to result/entire visit)

US\$0, US\$0.21, US\$3.50, US\$7 7 ART availability

Leaflet, phone call, text, in

5 Reasons to test person

4 Schedules/opening hours

Skilled, lay; self/partner; age; residence

Distribution/delivery strategies

Individual, batch; mobile, door- 3 Monetary Incentive to-door

Vaccines [31, 56-60, 63, 72, 73, 76, 94, 103, 104]

Product design

Efficacy

$50 \%, 70 \%, 95 \%, 99 \%$

9 Clades included

1,2

Yes/no

$1,5,20 \mathrm{~km}$

TB, blood pressure, diabetes mellitus, malaria

US\$0-0.85

Community event, pharmacy pick-up

Rapid, laboratory

In person, by phone

Alone, with partner/family

4, 12 weeks

1

Bacterial STI

1

20-90 min, 3 h

2

Immediate/no

Condom burst; instead of test 1 at clinic

Evenings, weekends, weekdays 1

US $\$ 3.50$ 
Table 2 (continued)

\begin{tabular}{|c|c|c|c|c|c|}
\hline Attribute & Level examples & $k$ & Attribute & Level examples & $\mathrm{k}$ \\
\hline Side effects & No, minor; aches, rash, fever & 9 & Vaccine-induced seropositivity & 3 months, 5 years; yes/no & 4 \\
\hline Duration of protection & 1 year, 10 years, lifetime & 7 & Formulation & Oral, injection & 4 \\
\hline Number doses & $1-5$ & 6 & Vaccine-induced infection & No, small chance & 1 \\
\hline \multicolumn{6}{|l|}{ Trial or service design } \\
\hline Cost & US\$3, US\$75, US\$250 & 8 & Free $T x$ if vaccine infected & Yes, no & 1 \\
\hline Location & Private, public hospital & 2 & Duration of trial & 3,5 years & 1 \\
\hline$\%$ of population vaccinated & Low, high & 2 & Compensation per visit & US\$25, US\$75 & 1 \\
\hline \multicolumn{6}{|c|}{ Topical microbicides $[25,36,62,67,69,85,105-107]$} \\
\hline \multicolumn{6}{|c|}{ Product design } \\
\hline Efficacy/effectiveness & $50 \%, 99 \%$ & 5 & Color & Clear, white, pearlescent, chalky & 2 \\
\hline Frequency of dosing & Daily, before/after sex & 4 & Messiness/wetness & No, some, a lot & 2 \\
\hline Side effects & $\begin{array}{l}\text { Irritation, itching, swelling, } \\
\text { burning }\end{array}$ & 4 & Shape & Bullet, tampon, tear drop, oval & 1 \\
\hline Pregnancy prevention & Yes/no & 4 & Gel texture & Watery, slippery, thick & 1 \\
\hline Partner awareness of use & No, maybe, leaves residue & 4 & Firmness & $250,2500,25,000$ & 1 \\
\hline Wait time after application & $10 \mathrm{~min}$ to $10 \mathrm{~h}$ before/after sex & 3 & Elasticity & Brittle, elastic & 1 \\
\hline Applicator & Yes/no; disposable/reusable & 3 & Duration of protection & $1-4$ h, 2-3 days & 1 \\
\hline STI prevention & Yes/no & 2 & Dosage (volume) & $1,2.5$ tablespoons & 1 \\
\hline Formulation & Gel, suppository & 2 & & & \\
\hline \multicolumn{6}{|l|}{ Service design } \\
\hline Cost & US\$0.30-7.60/dose & 5 & $\mathrm{Rx}$ requirement & Yes/no & 3 \\
\hline \multicolumn{6}{|c|}{ Voluntary medical male circumcision $[37-39,52]$} \\
\hline \multicolumn{6}{|l|}{ Service design } \\
\hline Staff/provider characteristics & Gender; informal/formal & 3 & Pain & 1 vs 4 days & 1 \\
\hline Waiting area characteristics & $\begin{array}{l}\text { Indoor/outdoor; age groups } \\
\text { separated }\end{array}$ & 2 & Counseling & Yes/no & 1 \\
\hline HIV testing & Required, opt-out, opt-in, none & 2 & Links to traditional MC schools & Yes/no & 1 \\
\hline Counseling characteristics & $\begin{array}{l}\text { Risk/benefits; with female } \\
\text { partner }\end{array}$ & 2 & Appointment style & Individual, groups & 1 \\
\hline Infection rate & $1 / 100,3 / 100$ & 1 & Schedule & Normal, extended/weekend & 1 \\
\hline Follow-up visit & Yes/no & 1 & Monetary incentive & None, lottery, transport voucher & 1 \\
\hline \multicolumn{6}{|c|}{ Prevention of mother-to-child transmission $[23,27,42,48,100]$} \\
\hline \multicolumn{6}{|l|}{ Product design } \\
\hline Risk of vertical transmission & $0,0.1,0.5,1 \%$ & 1 & Conception method & $\begin{array}{l}\text { Condomless sex with timed } \\
\text { ovulation }\end{array}$ & 1 \\
\hline Risk of horizontal transmission & $0,1,3,5 \%$ & 1 & Chance of conception per cycle & $5,10,25,60 \%$ & 1 \\
\hline \multicolumn{6}{|l|}{ Service design } \\
\hline Cost (per cycle; including travel) & US\$100-8000 & 2 & Provider attitude & Respectful, pleasant vs not & 1 \\
\hline Travel time to clinic & $30 \mathrm{~min}$ to $5 \mathrm{~h}$ & 1 & Other healthcare on site & Blood pressure, newborn care & 1 \\
\hline Location/facility type & Hospital, clinic, mobile & 1 & Support & Mothers group, counselor & 1 \\
\hline Husband/family involvement & Yes/no & 1 & & & \\
\hline
\end{tabular}

$A R T$ antiretroviral therapy, $H I V$ human immunodeficiency virus, $M C, S M S$ text message, $S T I$ sexually transmitted infection, $T B$ tuberculosis

ness to use condoms, and increase difficulties FSW already face in negotiating condom use [34].

\subsubsection{Voluntary Testing and Counseling}

Fifteen studies investigated combinations of voluntary testing and counseling product and service design [24, 28, 44, $45,50,53-55,86,88,93,95,96,99,102]$. 
3.2.2.1 Product Design The most common product attribute was fluid sampling method (oral fluid swab, finger prick blood, venipuncture blood). Generally, oral fluid-based sampling was preferred, then blood-based sampling through a finger prick, with venipuncture in the arm the least preferred. In Tanzania samples, however, oral fluid-based sampling was highly disliked, with preference for finger, then venous, blood collection $[44,45]$. One study found no significant difference in preferences for finger prick vs arm collection [55], and another found that participants were willing to accept a finger prick over an oral swab if it were cheaper, quicker, and also diagnosed other STIs [86]. One study considered STI testing along with HIV testing, with attributes of accuracy of syphilis results (potential for false positive) and the number of blood draws required (one or two). The number of blood draws mattered, but cost had the greatest impact [54].

3.2.2.2 Service Design The most common attribute was cost (US\$0-4 in lower income settings, US $\$ \leq 30$ in highincome settings); generally, participants preferred free testing $[28,50,54,55]$. Other common attributes involved logistical issues: location (home, clinic) and distance to services [28, 44, 50, 55]; evening or weekend hours [44]; length of the visit [28]; and time to get results [50, 54, 55]. Testing at home and immediate access to results were preferred. A closer distance mattered greatly in Tanzania, where it was more important than confidentiality and sampling method [44]. Two studies assessed alternatives to expand HIV testing: providing testing at dental offices (high acceptability of an oral test, but only for free [88]); and distributing tests at community events [53]. Multiple studies compared men and women, seeking strategies to increase men's access to HIV testing. These findings suggest more men may be reached with home-based, counselor-administered (vs nurse or self), immediate, and same-site access to antiretroviral therapy, bundling with other medical screenings (diabetes mellitus, tuberculosis), and providing monetary incentives [28, 45, 53].

3.2.2.3 Willingness Six studies assessed WTP for testing, or to be paid to test (as an incentive), or to use a free HIV test. Willingness to pay ranged from nothing (willingness to be tested for free only), to medians of US\$2-8 (lower income countries), and US\$25. Two studies assessed WTA testing in exchange for a payment. In the Tanzanian general population, the mean estimate was about US $\$ 3$, though there were differences by sex (men were willing to accept testing only at higher payments compared to women) and risk characteristics [99]. In Uganda, being paid to get tested influenced men, but not women, though both groups wanted free testing [28].

\subsubsection{HIV Self-Testing}

Fifteen studies examined HIV self-testing [29, 30, 32, 35, $41,47,68,75,77,80,82,84,89,97,98]$.

3.2.3.1 Product Design The most common product design attribute was sampling method (oral vs blood). The preferences were mixed, with some preferring oral sampling, and others finger prick blood sampling [30, 41, 47, 86]. Accuracy was also considered (99\% preferred over 95\%) [29, 84], the length of the "window period" when one would get a false negative [29, 77, 98], and combining STI testing in the same test cartridge [86].

3.2.3.2 Service Design The most common service design attribute was location (facility, mobile, home) [29, 30, 32, $35,41,47,75,84]$. Findings were mixed, with some preferring home-based testing and others clinic-based testing. Provider type (self vs community health worker) and characteristics (age, from the same or different village) [30, 32, 41, 89], and pre- and post-test counseling or support (by phone, leaflet, in person) also mattered, with some preferring tests conducted by or with providers or counselors, others comfortable with testing alone, and others indifferent [30, 41, $47,68,77]$. Cost mattered, with the strongest preference for free test kits [29, 30, 32, 41, 47, 75, 86]. In sub-group analyses, never-testers preferred oral over finger prick tests, while ever-testers were indifferent to type [30]. A study in MSM (UK) found two main classes of potential self-testers: the majority were comfortable with testing, and preferred provider-based testing; the minority, who had riskier sexual behaviors, preferred self-testing, and for free [29].

3.2.3.3 Willingness An additional seven studies investigated willingness/acceptability of self-testing. These studies found high willingness to use a self-test, including if offered at a dental office, mailed home, or when given a monetary incentive (any amount). Two studies examined subgroups, finding that both ever-testers and never-testers were willing to use self-tests, and that men, ever-testers, and people with higher incomes were more willing to use self-tests. One study examined WTP by group, finding that HIV testing clients were willing to pay more (US\$8.10) than MSM (US\$6.50) and FSW (US\$4.80). Dental clients were willing to pay US\$10-15, but not more [89]. Two studies also investigated if self-testers would return results to providers (only about half were willing, in one study) [80].

\subsubsection{Vaccine}

Thirteen studies investigated vaccines; nine were CAs on product design and design of hypothetical or actual randomized controlled trials $[31,56-60,63,72,103]$, and four 
on willingness to participate in those trials or pay for a future vaccine $[73,76,94,104]$.

3.2.4.1 Product Design Efficacy was in eight of nine studies and was the most important attribute; high efficacy (95-99\%) was always in the highest rated vaccine profile [31, 57-60, 63, 72, 94, 103]. Side effects was a common attribute; lack of side effects was rated highly across studies [31, 56-60, 63, 94, 103]. Several included the risk of vaccine-induced seropositivity (VISP) [false-positive result due to the vaccine], or length of time of VISP (months, years); not experiencing VISP was highly rated [31, 56, 59, 103], and was more important than efficacy in one study [59]. One study assessed the risk of vaccine-induced HIV infection (true positive result), and this was the most important attribute in that study [56]. Duration of protection was also common (10 years vs lifetime; longer preferred) [31, 58-60, $63,94,103]$; number of doses (one to four; fewer weakly preferred) [56-58, 60, 63, 103]; and formulation (oral vs injection; not significant) [57, 58, 60, 63].

3.2.4.2 Trial and Service Design Studies investigated location of administration [31, 59], proportion of population already vaccinated [31, 59], duration of trial [56], and reimbursement amount for trial participation, but these were not strongly rated either way [56]. Availability of free treatment if seroconversion occurs because of trial participation was important, but less so than vaccine-induced infection and VISP [56]. A few studies also assessed preferences around single or multiple strains (clades) protected against in a vaccine (results mixed) [57, 58, 63, 103], and the cost of a future vaccine (price mattered less compared to other attributes) $[31,57-60,63,72,103]$.

3.2.4.3 Willingness Four studies investigated willingness to participate in a vaccine trial or to pay for a vaccine [73, $76,94,104]$. One study asked subjects on their willingness to participate at four timepoints leading up to an actual trial; greater stated WTP predicted later enrollment [76]. In two CV studies, respondents in Mexico were willing to pay around US $\$ 4670$ for a vaccine with lifetime protection, and respondents in Thailand were willing to pay US\$610-809 depending on effectiveness $(50 \%$ vs $95 \%)$ [94, 104].

\subsubsection{Topical Microbicides}

Nine studies investigated vaginal or rectal topical microbicides [25, 36, 62, 67, 69, 85, 105-107]. (One was on intravaginal devices, and included microbicides [36].)

3.2.5.1 Product Design Attributes included efficacy [36, $62,105,106]$; side effects [25, 62, 69, 106]; frequency of use/dosing [105, 106]; additional prevention from preg- nancy and/or STI [36, 67, 69]; and potential for partner awareness of use (e.g., leaves residue on partner) [25, 36, 67]. Additional attributes were wait time between application to coitus (minutes, hours) [67, 106, 107], using an applicator (yes vs no, reusable vs disposable) [25, 106], and formulation (including shape, color, consistency) [25, 36, $62,67,85,105,107]$. Summarizing across studies, participants would prefer a highly efficacious product that used an applicator, did not require daily use, had no side effects, had shorter wait times after application, prevented pregnancy, and could be used covertly from partners. A highly effective product was important to participants, but there was some willingness to trade lower efficacy for other desirable attributes (e.g., no side effects).

3.2.5.2 Service Design The only service-oriented attributes for microbicides were cost (US\$0.30-7.60 per dose) $[25,36$, $62,105]$ and requiring a prescription vs over-the-counter availability $[62,105,106]$. Participants generally preferred a lower cost per dose (US\$0.30), but one study found a greater WTP for high effectiveness, and a moderate amount more for a gel over a suppository and for intermittent vs daily use [105]. Findings on prescription vs over-the-counter were mixed across studies.

\subsubsection{VMMC}

Four studies investigated VMMC; three in South Africa were on different aspects of the same program, and the other was in Tanzania [37-39, 52]. None investigated product design (for example, PrePex device), but rather investigated service design [37, 38, 52] and motivations for accessing VMMC and risk compensation ("condom avoidance") [39].

3.2.6.1 Service Design Attributes in both the Tanzanian and South African studies were provider characteristics (e.g., sex), waiting area characteristics (e.g., separation of younger boys and grown men), and HIV testing requirements (opt-out, opt-in) [37, 52]. In South Africa, follow-up visit, low infection rate, less post-operative pain, and outside waiting areas were important [37]. One South African study evaluated preferences for promotion of a VMMC program. Participants preferred television marketing, support from church/school leaders, and endorsement by a national program [38]. In Tanzania, men preferred separate waiting areas for age groups, opt-out HIV testing, male-only service providers, counseling for female partners, and a monetary incentive in the form of a transport voucher vs a lottery [52].

3.2.6.2 Risk Compensation Last, one of the studies in South Africa investigated if risk reduction ("reduces cervical cancer risk in women; reduces STI risk, reduces HIV risk"); risk compensation ("no condom needed"); or other features 
of VMMC "(improves hygiene, more enjoyable sex") were motivators for accessing the service. This study found that risk reduction effects were the strongest, with no evidence of being motivated by condom avoidance among Black and Coloured populations, but evidence of this among White South Africans [39].

\subsubsection{PMTCT}

There were five studies that considered PMTCT; two of these evaluated product and service design attributes, one willingness. Two investigated motivations to seek PMTCT services $[23,27,42,48,100]$.

3.2.7.1 Product Design One study investigated product design of PMTCT, including aspects of safer conception (which can be technology assisted). This considered both serodiscordant and seroconcordant couples' preferences for risk of vertical transmission (to the child) and horizontal transmission (to the partner), conception method, and chance of conception per cycle. Serodiscordant couples (female HIV positive, male negative) expressed decreased utility with risk of any transmission, and preferred home insemination with a syringe to other conception methods. Seroconcordant couples expressed decreased utility with risk of vertical transmission; they preferred clinic-based conception methods over other methods (e.g., condomless sex timed with ovulation) [48].

3.2.7.2 Service Design Two studies considered cost, which was important to participants in both [23, 42]. Trust in provider [23] and respectful attitude of provider were strongly preferred attributes, with provider attitude two to three times more important than other attributes [42]. Co-location of other healthcare services was also important, but facility type did not influence participants [42].

3.2.7.3 Willingness One study investigated willingness to accept or be paid for informal care around PMTCT in Malawi. Willingness to pay for such services was US $\$ 13$, while willingness to accept payment to perform such services was US\$30 [100].

3.2.7.4 Motivators/Values Two studies evaluated tradeoffs of motivations or priorities placed on preventing HIV in participants' partners and/or children. These included statements such as antiretroviral therapy "keeps me healthy for my family," "prevents me form getting sick from infections," and "prevents infant's illness." Both studies compared participants in antepartum and postpartum periods, finding slightly different priorities. Living a long life was more highly rated during the antepartum period, while keeping CD4 count high was more important postpartum.
However, trust in providers was highly prioritized in both periods [23, 27].

\subsubsection{Other/Multiple Technologies}

Four studies fell outside of HIV prevention technology categories summarized above (not in Table 2). One investigated WTP for condoms. It found that people who access free condoms in in East and Southern Africa were willing to pay for them, even more than the average cost in their countries, and that brand mattered [92]. Another found that women in the USA were willing to try new types of condoms and lubricants if they increased their sexual pleasure [81]. The only study that considered healthcare workers' prevention needs investigated WTP for a needle-stick prevention device [74]. Last, one study compared WTA various HIV prevention modalities (topical microbicides, VMMC, PrEP, vaccine, and external and internal condoms) pre- and post-intervention, an educational presentation on prevention options. External condoms were preferred both preand post-intervention, but PrEP, microbicides, VMMC, and internal condoms were more highly rated post-intervention [87].

\subsection{Quality Assessment}

The mean PREFS score was 3.47/5 (standard deviation: 0.81 ; range $2-5$ ) [Table 1 ]. Nearly all studies $(86 \%)$ included their preference imperative in their explanation of the purpose of the study ("purpose"). Only $14 \%$ of studies included an explanation of how the respondents varied from nonrespondents ("respondents"). Almost all (99\%) sufficiently explained the preference assessment method ("explanation"), $60 \%$ of studies met criteria for reporting their findings ("inndings") and 89\% used appropriate statistical tests ("significance").

The mean stars awarded for our adapted version of NOS was 4.5/8 (standard deviation $=2.1$, range $2-8)($ Table 1$)$. For representativeness, $89 \%$ of studies received at least one star. For heterogeneity analysis, $82 \%$ of studies received a least one star, and $55 \%$ of studies received two stars. All the studies received at least one star for exposure assessment, and $43 \%$ earned two stars. Only $27 \%$ of studies justified the sample size. Furthermore, also captured by PREFS, only $14 \%$ of studies included an explanation of how the respondents varied from non-respondents and $89 \%$ used appropriate statistical tests.

Publication year was not significantly associated with either having a high- or low-quality PREFS $(p=0.68)$ or NOS score $(p=0.64)$. The proportion of high- and lowquality studies as measured using either PREFS or NOS did not vary among BWS, CA, CV, WTP, or WTA studies (all $p>0.05$ ). Studies using DCE were more likely to 
have a high-quality PREFS score $(p=0.049)$ and NOS score $(p=0.001)$ as compared to other methods.

\section{Discussion}

This systematic review summarizes the application of quantitative preference elicitation techniques to measure preferences for HIV prevention technologies. With 84 included studies covering a range of methods and prevention technologies, this review provides a synthesis of how these methods are used and applied in HIV prevention, trends in attribute identification, survey development, and analysis, as well as findings for HIV prevention program implementers. Next, we present some key findings and situate these in the literature.

Our study overlaps with another recent systematic review by Humphrey et al. [13]. That systematic review assessed preference elicitation methods applied to healthcare workers' preferences and priorities in SSA, and included 77 articles, more than half of which used DCEs [14]. The present study differs from that review in important ways. First, Humphrey et al. limited the preference methods to DCE, CA, and BWS, while the present study had broader inclusion of elicitation methods. Second, our study specifically targeted HIV prevention technologies (e.g., PrEP, self-testing devices, vaccines), while Humphrey et al.'s review included any HIV-related topic, including prevention, service delivery, and antiretroviral therapy for those living with HIV. Thus, while several studies were included in both reviews $(18,21 \%$ of the 84 in this review), each review presents largely unique findings.

\subsection{Preference Elicitation Methods}

From the list of 22 eligible preference elicitation techniques, only six types were employed in the included studies (CA, DCE, WTA, CV, WTP, and BWS). Three techniques (CA, DCE, and WTA) accounted for nearly $80 \%$ of the included studies. There may be trends in the use of these techniques over time. For example, CA appeared only after $2005,95 \%$ of DCEs were conducted after 2013, and $90 \%$ of the most recent ten studies used DCEs.

Classifying studies needed careful consideration and discussion between authors. For example, one study did not use any of the pre-determined labels, rather the term "choice elicitation tasks" [105]; for the purposes of this review, we labeled this BSW (case 3). Further, we re-classified some studies that were published as CA. Upon examination of their methods, we labeled them as DCE for this review, given their use of choice tasks comparing two or more profiles [27, 37-40]. "True" CA studies involved rating or ranking of profiles individually, rather than in head-to-head comparisons.
Conjoint analysis has been used as an umbrella term when referring to DCE or other choice-based methods, but they are distinct methods with disparate underlying theoretical and statistical models [18].

Best-worst scaling techniques were employed in only two papers $[23,105]$. The lack of BWS in the included studies may be because BWS is commonly used to understand priorities, while this review focused on trade-offs for specific technologies. Although DCEs can be used to generate some information about the relative importance of attributes, large variation in the calculation and context of attribute importance for DCEs complicates the interpretation of these data [108]. Indeed, the two BWS studies did focus on priorities about a prevention technology (PMTCT) rather than product or service characteristics per se. For most of the included studies, the objective was not to do exploratory work understanding priorities but rather to quantify preferences for hypothetical or actual prevention modalities' characteristics. A takeaway message is that the field within health and medicine is growing, but investigators are not yet utilizing all the possible preference elicitation tools available. For example, it may be that $\mathrm{CA}$ gained rather than lost popularity in HIV preference research, and the use of DCE is growing, but other preference elicitation tools such as BWS and direct elicitation approaches may be useful and less complicated for both respondents and researchers to design and analyze [20].

An interesting finding of the current study was the relatively low utilization of rigorous and largely qualitative survey development/attribute identification methods. Fewer than half of studies reported using any of the established methods of survey development including literature review, qualitative/formative interviews, expert consultation, pretesting, and pilot testing [26]. There is growing pressure in the patient preference community to develop and apply standards to inform the qualitative development of patient preference studies [109]. We would add that in addition to using qualitative methods to inform the development of such studies, authors should also be transparent in their reporting of the survey development methods used, and that this should perhaps be an additional item for a future quality assessment tool in preference studies.

\subsection{HIV Prevention Technologies}

This review included HIV prevention technologies that were either currently available or in various stages of pre-market development. While a broad range of technologies were eligible for inclusion, the included studies measured preferences for PrEP, HIV testing, vaccines, topical microbicides, VMMC, PMTCT, and condoms. The hypothetical nature of the product varied and the included studies measured preferences for technologies already readily available (condoms); 
available in some countries but not necessarily where the study was conducted (oral PrEP, HIV self-testing); available in the study location (VMMC, oral PrEP); or in the research pipeline (injectable PrEP, vaccine). With such diversity, there were also different types of attributes that the participants had to consider. We grouped these broadly under product design (e.g., side effects, modality, shape) and service design (e.g., cost, distance, provider type), with some studies examining a combination of these. This synthesis may be useful to both preference researchers designing preference surveys and HIV prevention implementers seeking to design programs for maximum uptake.

The HIV technologies investigated in the included studies were largely focused on PrEP and HIV testing, perhaps reflecting trends in HIV funding and research priorities [110]. Pre-exposure prophylaxis, for example, in its many modalities, such as the oral pill, long-acting injectable, intravaginal device, or topical microbicide gel, accounted for one in three of the studies. Pre-exposure prophylaxis is the newest of the technologies, and the first biomedical intervention for sexual prevention of HIV since condoms. Despite being approved for use in the USA since 2012, uptake has been low, and persistence lower still [111]. Alternative formulations and modalities of PrEP hold promise for overcoming some of the challenges with PrEP as a daily pill, such as adherence and persistence $[112,113]$. Preference elicitation methods are well suited to understanding user preferences for PrEP, and these findings could be used to inform tailored interventions.

The included studies indicated heterogeneity in PrEP modality preferences, with some favoring the daily pill, others on-demand gel, and others injectable forms. This is in line with findings from multi-country studies of actual uses of various PrEP products [114]. The vaginal ring was not preferred in any of the studies that investigated it compared to other formulations. However, in studies where women tried various products over months, including the ring, there was comfortability and acceptability with the ring $[114,115]$. This suggests that users may need to try and gain familiarity with such novel products, rather than rate them hypothetically.

Traditional testing and HIV self-testing accounted for another third of included studies. This reflects (1) user preferences and acceptability around testing through blood or saliva (findings mixed, with some preferring finger prick blood and some the saliva), and (2) alternative implementation strategies to increase testing, particularly among men in SSA (e.g., home and community-based testing; evening and weekend hours). Men in SSA have been less likely to access HIV-related services, are diagnosed later, and more likely to die even on antiretroviral therapy, compared to women [116, 117]. Thus, it is important to identify strategies that better meet their needs, as well as the needs of other populations at high risk.

Articles on preferences for future vaccines and actual and hypothetical vaccine trials were moderately common, though none were published since 2013. Two HIV vaccine trials are ongoing, and one was stopped early 2020, showing no preventive effect [118]. Prevention of mother-to-child transmission, VMMC, and condoms accounted for most of the remaining third, all aimed at understanding user preferences of existing services to adapt services in hopes of boosting uptake. Only one study focused on HIV prevention among healthcare workers, investigating WTP for a needlestick prevention device. No studies investigated PEP or substance use harm reduction technologies (syringe exchange, MAT).

In the included studies, about two-thirds of participants were men and one-third were women. Gay and bisexual men and other MSM are at an elevated risk of HIV globally [119], and many of the included studies were among these groups. Many of the included women were young and adolescent women, who are at a particularly high risk in some settings (Eastern and Southern Africa), and many others were engaged in sex work, another highly vulnerable group, depending on context [120]. A tiny proportion $(0.4 \%)$ of the included participants were transgender. This is despite the fact that transgender women who have sex with men have a 49 times higher odds of living with HIV than other adults of reproductive age, globally [121]. No studies reported including transgender MSM, another at-risk, but largely invisible, group [122, 123], nor other gender-diverse identities (i.e., non-binary people).

People who use drugs were included in only six studies [57, 58, 65, 70, 76, 93], and no drug use-related HIV prevention technologies were examined (e.g., syringe exchange). Multiple studies were conducted among general populations not at risk of HIV. Investigators should recruit from the populations most likely to use and benefit from the technology or service, should it become available, to uphold the principle of justice in human subject research and ensure the findings are relevant to people most in need of HIV prevention. There was limited geographic representation in the included studies. Nearly $90 \%$ were in North America and SSA, largely the USA and South Africa. No studies were conducted in Australia, Oceania, or Middle East/North Africa, and few throughout Latin America and the Caribbean, Asia, and Europe. This may be reflective of the researchers' and funders' priorities, as most funding was also from US funding institutions. The USA funds a large amount of HIV-related research and programming in SSA through PEPFAR, given the high HIV burden [124]. 


\subsection{Quality Review}

The high endorsement we observed across many of the quality metrics used in this study (e.g. 90\% representativeness, 90\% significance) indicates a need for quality assessment tools that can better distinguish high- from low-quality studies in preference contexts. That only $14 \%$ of studies included an appropriate assessment of how study respondents compared to non-respondents is a significant concern to the external validity of these studies. Though the PREFS checklist is a critical appraisal tool for use in the evaluation of stated-preference surveys [22], going into this review we were aware of existing shortcomings of the checklist. These shortcomings include a lack of discrimination across studies and the absence of important quality criteria that have been refined by the US Food and Drug Administration, including patient centeredness and heterogeneity [125]. Adding the NOS measures strengthened the quality appraisal by allowing for the addition of domains such as sample size justification and representativeness of the sample. However, other important criteria are still missing from preference quality appraisal tools, such as reporting on potential scale differences between samples, patient centricity and relevance of the preference-elicitation questions, and rigor of the instrument development processes.

\subsubsection{Limitations}

This review has limitations. There is not a definitive list of quantitative preference elicitation methods and we would expect variation in the return of results based on the methods we included. For instance, ranking and rating methods were omitted here as they were not explicitly included in Soekhai and colleagues' list [17]. However, rating and ranking are included in other prominent lists for preferences, including the Medical Device Innovation Consortium Appendix on preference methods [126]. Including those methods would have likely included more articles, and perhaps a wider variety of HIV prevention methods. Given this review's focus on quantitative preference elicitation methods, qualitative studies that examined HIV prevention preferences were excluded. Such studies nevertheless provide valuable information about users' preferences and a more in-depth understanding of facilitators and barriers to product use but were beyond the scope of this review. This review also only included peer-reviewed literature. Thus, we may have missed studies that were programmatically focused and only in the gray literature, and may have a publication bias. The quality review of this study used the pre-exiting NOS for cross-sectional studies, adapting it for relevance to preference studies. However, this adaptation has not been validated and warrants further exploration of its usefulness.
This review summarizes the quantitative preference elicitation methods, attribute identification approaches, and statistical analyses used to measure preferences for HIV prevention technologies globally. While this literature illuminates preferences and may guide product development and adaptation of services, the extent to which such preference studies impact actual services and products is not well known and is an area for future research. Additionally, future work should continue to develop more rigorous quality assessment tools for use in reporting on stated-preference studies.

\section{Conclusions}

Human immunodeficiency virus prevention options continue to expand to offer a "menu" of biomedical options that can better meet the needs of disparate communities. However, these technologies only work if people at risk for HIV utilize them during periods of risk; and we know from low levels of condom use that simply because a method works, does not mean people will use it. Patient-centered preference elicitation methods can gather information about product and service characteristics that may then be used to influence uptake of HIV prevention methods. This review synthesizes extant literature on these topics and can provide practictioners with important information about preferences that may be used to improve prevention products and services. These methods, packaged together in combination prevention interventions [3] that are tailored to different community needs, will ultimately reduce HIV incidence.

\section{Declarations}

Funding This work was supported by the National Institutes of Health, grant number K01MH114715 (Beckham).

Conflicts of interest/competing interests $\mathrm{S}$ Wilson Beckham receives research funding from Viiv Healthcare. Norah L. Crossnohere, Margaret Gross, and John F.P. Bridges have no conflicts of interests that are directly relevant to the content of this article.

Ethics approval Not applicable.

Consent to participate Not applicable.

Consent for publication Not applicable.

Availability of data and material Search terms for the systematic review are provided in the Appendix.

Code availability Not applicable.

Authors' contributions SWB and JFPB conceived of and planned the review. SWB wrote the protocol. SWB and NLC wrote the manuscript with direction from JFPB. MG wrote the search terms and ran the searches. SWB and NLC screened titles and abstracts. SWB and NLC 
reviewed full texts. SWB abstracted data. NLC conducted the quality review.

Open Access This article is licensed under a Creative Commons Attribution-NonCommercial 4.0 International License, which permits any non-commercial use, sharing, adaptation, distribution and reproduction in any medium or format, as long as you give appropriate credit to the original author(s) and the source, provide a link to the Creative Commons licence, and indicate if changes were made. The images or other third party material in this article are included in the article's Creative Commons licence, unless indicated otherwise in a credit line to the material. If material is not included in the article's Creative Commons licence and your intended use is not permitted by statutory regulation or exceeds the permitted use, you will need to obtain permission directly from the copyright holder. To view a copy of this licence, visit http://creativecommons.org/licenses/by-nc/4.0/.

\section{Appendix A: Search terms}

\section{PubMed}

\section{Concept 1: HIV}

"HIV"[mesh] OR "HIV"[tw] OR “AIDS"[tw] OR "HIV-1"[mesh] OR "HIV-2"[mesh] OR "Human immunodeficiency viruses"[tw] OR "HTLV-III"[tw] OR "Human Immunodeficiency Virus"[tw] OR "Acquired Immune Deficiency" [tw] OR “Acquired Immunodeficiency Syndrome"[Mesh] OR “Acquired Immunodeficiency"[tw] OR “HIV Infections"[Mesh] OR "T Lymphotropic Virus Type III Infections”[tw] OR “T-Lymphotropic Virus Type III Infection" [tw] OR “acquired immunologic deficiency”[tw]

\section{AND}

\section{Concept 2: Prevention}

"Pre-Exposure Prophylaxis"[Mesh] OR "Pre Exposure Prophylaxis"[tw] OR "Preexposure Prophylaxis"[tw] OR “prep"[tw] OR “emtricitabine”[tw] OR “emtricitabine" [Supplementary Concept] OR "Emtriva"[tw] OR "Coviracil"[tw] OR "Truvada" [tw] OR "51sx" [tw] OR “Antiretroviral Prophylaxis”[tw] OR “Tenofovir”[Mesh] OR “tenofovir”[all fields] OR “9-(2-Phosphonylmethoxypropyl) adenine"[tw] OR "Viread"[tw] OR "Elvitegravir, Cobicistat, Emtricitabine, Tenofovir Disoproxil Fumarate Drug Combination"[Mesh] OR “Stribild”[tw] OR “quad"'[tw] OR “Cobicistat”[Mesh] OR “Cobicistat"[tw] OR “Tybost”[tw] OR “GS 9350” [tw] OR “Emtricitabine, Rilpivirine, Tenofovir Drug Combination"[Mesh] OR "Rilpivirine”[Mesh] OR "Rilpivirine"[tw] OR "Emtricitabine"[Mesh] OR "Emtricitabine"[tw] OR "Emtriva"[tw] OR “Coviracil"[tw] OR "emtricitabine tenofovir alafenamide" [Supplementary Concept] OR “Descovy”[tw] OR FTC[tw] OR TDF[tw] OR TDF[tw] OR “Anti-Retroviral Agents”[Mesh] OR “Anti-Retroviral Agents"[Pharmacological Action] OR "anti retrovirus agent" [tw] OR “anti retrovirus agents" [tw] OR “antiretrovirus agent"[tw] OR “antiretrovirus agents"[tw] OR “ART”[tw] OR “anti retroviral”[tw] OR “antiretroviral”[tw]
OR “Antiretroviral Therapy, Highly Active"[MeSH] OR “HAART”[tw] OR "HIV Prevention”[tw] OR “HIV medicine"[tw] OR "HIV medication"[tw] OR "HIV medications"[tw] OR "condom"[tw] OR "condoms"[tw] OR “self testing”[tw] OR "self evaluation"[tw] OR VMMC[tw] OR "voluntary male medical circumcision”[tw] OR “voluntary medical male circumcision" [tw] OR "Circumcision, Male"[Mesh] OR "male circumcision”[tw] OR "Condoms"[Mesh] OR “Condoms, Female"[Mesh] OR bictegravir[tw] OR racivir[tw] OR "self-testing”[tw] OR “self-test”[tw] OR “oral HIV test”[tw] OR "HIV oral test"[tw] OR TMC278LA[tw] OR cabotegravir[tw] OR "long-acting”[tw] OR "zinc carrageenan"[tw] OR "zinc gel”[tw] OR “cervical ring”[tw] OR “Nonoxynol-9”[tw] OR “Cellulose sulfate"[tw] OR "Pro 2000”[tw] OR "Buffer gel”[tw] OR “Carraguard"[tw] OR "Savvy”[tw] OR" Cellulose acetate phthalate"[tw] OR "Dapivirine"[tw] OR (MIV 150[tw] AND “zinc acetate"[tw] AND carrageenan[tw]) OR "Maraviroc"[tw] OR “IQP-0528”[tw] OR "DS003”[tw] OR "Lactin V"[tw] OR “Mucocept"[tw] OR "Maraviroc"[tw] OR “Pyrimidine-diones"[tw] OR "Harm Reduction"[Mesh] OR “Harm Reduction”[tw] OR "Harm Minimization”[tw] OR "Needle-Exchange Programs"[Mesh] OR "Needle-Exchange Programs"[tw] OR "Needle-Exchange Program"[tw] OR "Needle-Exchange Programme"[tw] OR "Needle-Exchange Programme"[tw] OR "Syringe Exchange Programs"[tw] OR "Syringe Exchange Programmes"[tw] OR "Syringe Exchange Programme"[tw] OR "Syringe Exchange Program"[tw] OR "Supervised Injecting Center" [tw] OR “Supervised Injecting Centers"[tw] OR "Supervised Injecting Centre"[tw] OR "Supervised Injecting Centres"[tw] OR “Supervised Injecting Facilities”[tw] OR "Supervised Injecting Facility”[tw]

\section{AND}

\section{Concept 3: Stated Preference}

stated-preference*[tw] OR stated-choice*[tw] OR "Patient weighting"[tw] OR "Patient rating"[tw] OR "Patient ranking"[tw] OR "Patient perspective"[tw] OR "Patient priorities"[tw] OR "Patient preference"[MeSH] OR "Patient Preference"[tw] OR "Patient Preferences" [tw] OR "Preference-based approach"[tw] OR "Preference-based approaches"[tw] OR "Preference-based method"[tw] OR "Preference-based methods"[tw] OR trade-off*[tw] OR compositional[tw] OR decompositional[tw] OR rank*[tw] OR "best-worst"[tw] OR worst-best[tw] OR "object scaling"[tw] OR BWS[tw] OR WBS[tw] OR "maxdiff scaling"[tw] OR "maximum difference" [tw] OR maxdiff[tw] OR “max diff”' $t w]$ OR “discrete choice"[tw] OR “DCE”[tw] OR conjoint analys*[tw] OR allocation of point*[tw] OR "point allocation*"[tw] OR budget allocation*[tw] OR magnitude estimation*[tw] OR pairwise choice*[tw] OR paired comparison*[tw] OR self-explicated method*[tw] 
OR self-explicat*[tw] OR self explicat*[tw] OR "direct assessment" [tw] OR "direct preference assessment" [tw]

\section{Appendix B: Quality Assessment}

Adapted Newcastle-Ottawa Scale for use in patient preference studies ${ }^{\mathrm{a}}$ Representativeness of the sample

*A

$* \mathrm{~B}$

$\mathrm{C}$

$\mathrm{D}$

Sample size

$* \mathrm{~A}$

B

Non-respondents

$* \mathrm{~A}$

C

Validity of the preference elicitation

**A

$* \mathrm{~B}$

$\mathrm{C}$

Heterogeneity: Potential differences between respondent groups have been explored

*A
Truly representative of the average in the target population (all subjects or random sampling)

Somewhat representative of the average in the target population (non-random sampling)

Selected group of users

No description of the sampling strategy

Justified and satisfactory (based on power calculations or feasibility)

Not justified

Comparability between respondents and non-respondent characteristics is established, and response rate is satisfactory

Response rate is unsatisfactory, or comparability between respondents and non-respondents is unsatisfactory

No description of response rate or the characteristics of the respondents and non-respondents as defined by using an instrument that has been previously evaluated in the current setting or for which there is a clearly explained and rigorous instru-

Non-valid measurement tool, but the tool is available or described by authors

No description of the measurement tool

Study includes one aspect of respondent heterogeneity and its effect on preferences
Validated measurement tool, ment development process

\section{Representativeness of the sample}

*A Truly representative of the average in the target population (all subjects or random sampling)

$* \mathrm{~B}$

Study includes two or more aspects of respondent heterogeneity and its effect on preferences

\section{Statistical test}

*A

The statistical test used to analyze the data is clearly described and appropriate, and the measurement of the association is presented, including confidence intervals and the probability $(p$ value)

B

The statistical test is not appropriate, not described, or incomplete

${ }^{\text {a } A d a p t e d ~ f r o m ~ M o d e s t i ~ e t ~ a l . ~[16] ~}$

\section{Appendix C: Quality review (PREFS) vs Newcastle-Ottawa Scale (NOS) scatterplot}

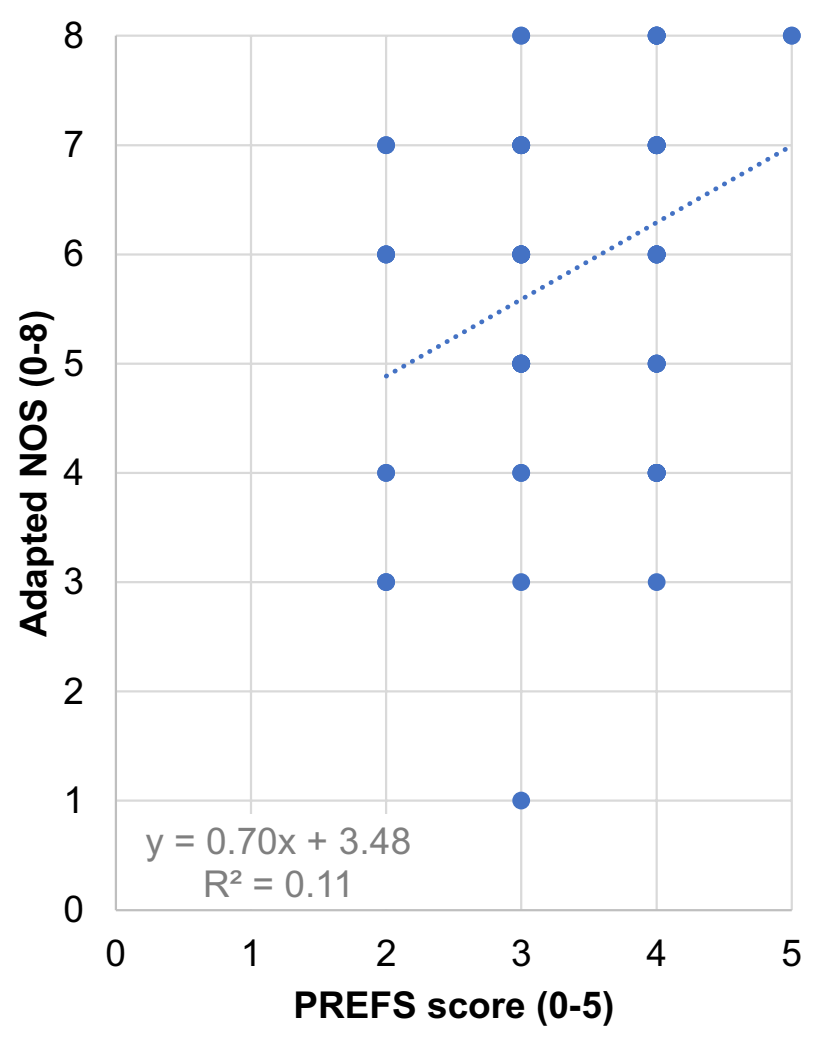




\section{References}

1. Centers for Disease Control. Advancing HIV prevention: new strategies for a changing epidemic: United States, 2003. MMWR Morb Mortal Wkly Rep. 2003;52(15):329-32.

2. Karki P, et al. The impact of methadone maintenance treatment on HIV risk behaviors among high-risk injection drug users: a systematic review. Evid Based Med Public Health. 2016;2:e1229.

3. Padian NS, et al. HIV prevention transformed: the new prevention research agenda. Lancet. 2011;378(9787):269-78.

4. Fonner VA, et al. Effectiveness and safety of oral HIV preexposure prophylaxis for all populations. AIDS. 2016;30(12):1973-83.

5. US Food and Drug Administration. FDA approves second drug to prevent HIV infection as part of ongoing efforts to end the HIV epidemic. 2019. https://www.fda.gov/news-events/press-annou ncements/fda-approves-second-drug-prevent-hiv-infection-partongoing-efforts-end-hiv-epidemic. Accessed 13 Aug 2020.

6. Cohen MS. Prevention of HIV-1 infection with early antiretroviral therapy. N Engl J Med. 2011;365(6):493-505.

7. Gardner M, et al. The spectrum of engagement in HIV care and its relevance to test-and-treat strategies for prevention of HIV infection. Clin Infect Dis. 2011;52(6):793-800.

8. McNairy ML, El-Sadr WM. Antiretroviral therapy for the prevention of HIV transmission: what will it take? Clin Infect Dis. 2014;58(7):1003-11.

9. Baeten JM, et al. Use of a vaginal ring containing dapivirine for HIV-1 prevention in women. $\mathrm{N}$ Engl $\mathrm{J}$ Med. 2016;75(22):2121-32.

10. HIV.gov. Long-acting HIV prevention tools. 2019. Available from: https://www.hiv.gov/hiv-basics/hiv-prevention/potentialfuture-options/long-acting-prep. Accessed 28 Nov 2020.

11. HIV Prevention Trials Network (HPTN). HPTN 083 Study demonstrates superiority of cabotegravir for the prevention of HIV. 2020. Available from: https://www.hptn.org/news-andevents/press-releases/hptn-083-study-demonstrates-superiorit y-cabotegravir-prevention-hiv?utm_source=IAS\&utm_campa ign=3b6214676e-daily-delegate-11-July\&utm_medium=email \&utm_term=0_58c4aa5b50-3b6214676e-103223869. Accessed 28 Nov 2020.

12. Brady M, Manning J. Lessons from reproductive health to inform multipurpose prevention technologies: don't reinvent the wheel. Antiviral Res. 2013;100(Suppl.): S25-31.

13. Humphrey JM, et al. Stated-preference research in HIV: a scoping review. PLoS One. 2019;14(10):e0224566.

14. Brown L, et al. Applying stated-preference methods to improve health systems in sub-Saharan Africa: a systematic review. Expert Rev Pharmacoecon Outcomes Res. 2017;17(5):441-58.

15. Bridges J, et al. Patient preference methods: a patient centered evaluation paradigm. ISPOR Connect. 2007;13(6):4-7.

16. Modesti PA, et al. Panethnic differences in blood pressure in Europe: a systematic review and meta-analysis. PLoS One. 2016;11(1):e0147601.

17. Soekhai V, et al. Compendium of methods for measuring patient preferences in medical treatment. Value Health. 2017;20(A399-A811):A684-5.

18. Louviere JJ, Flynn TN, Carson RT. Discrete choice experiments are not conjoint analysis. J Choice Model. 2010;3(3):57-72.

19. Bien DR, et al. Patients' preferences for outcome, process and cost attributes in cancer treatment: a systematic review of discrete choice experiments. Patient. 2017;10(5):553-65.

20. Cheung KL, et al. Using best-worst scaling to investigate preferences in health care. Pharmacoeconomics. 2016;34(12):1195-209.
21. Showalter TN, Mishra MV, Bridges JF. Factors that influence patient preferences for prostate cancer management options: a systematic review. Patient Prefer Adherence. 2015;9:899-911.

22. Joy SM, et al. Patient preferences for the treatment of type 2 diabetes: a scoping review. Pharmacoeconomics. 2013;31(10):877-92.

23. Kim HY, et al. Maternal priorities for preventive therapy among HIV-positive pregnant women before and after delivery in South Africa: a best-worst scaling survey. J Int AIDS Soc. 2018;21(7):e25143.

24. Nguyen LH, et al. Mobilization for HIV voluntary counseling and testing services in Vietnam: clients' risk behaviors, attitudes and willingness to pay. AIDS Behav. 2016;20(4):848-58.

25. Hardy E, et al. Women's preferences for vaginal antimicrobial contraceptives IV. Attributes of a formulation that would protect from STD/AIDS. Contraception. 1998;58(4):251-5.

26. Bridges JF, et al. Conjoint analysis applications in health: a checklist: a report of the ISPOR Good Research Practices for Conjoint Analysis Task Force. Value Health. 2011;14(4):403-13.

27. Kim HY, et al. Maternal motivation to take preventive therapy in antepartum and postpartum among HIV-positive pregnant women in South Africa: a choice experiment. AIDS Behav. 2019;23(7):1689-97.

28. Korte JE, et al. HIV testing preferences among pregnant women attending antenatal care and their male partners: a discrete choice experiment in Uganda. Afr J AIDS Res. 2019;18(4):332-40.

29. Miners A, et al. Preferences for HIV testing services among men who have sex with men in the UK: a discrete choice experiment. PLoS Med. 2019;16(4):e1002779.

30. Strauss M, et al. HIV testing preferences among long distance truck drivers in Kenya: a discrete choice experiment. AIDS Care. 2018;30(1):72-80.

31. Cameron MP, et al. The marginal willingness-to-pay for attributes of a hypothetical HIV vaccine. Vaccine. 2013;31(36):3712-7.

32. Indravudh PP, et al. 'I will choose when to test, where i want to test': investigating young people's preferences for HIV selftesting in Malawi and Zimbabwe. AIDS. 2017;31:S203-12.

33. Kuteesa MO, et al. Acceptability and predictors of uptake of anti-retroviral pre-exposure prophylaxis (PrEP) among fishing communities in Uganda: a cross-sectional discrete choice experiment survey. AIDS Behav. 2019;23(10):2674-86.

34. Quaife M, et al. The effect of HIV prevention products on incentives to supply condomless commercial sex among female sex workers in South Africa. Health Econ. 2018;27(10):1550-66.

35. Sibanda EL, et al. Applying user preferences to optimize the contribution of HIV self-testing to reaching the "first 90" target of UNAIDS Fast-track strategy: results from discrete choice experiments in Zimbabwe. J Int AIDS Soc. 2019;22(Suppl. 1):e25245.

36. Terris-Prestholt F, et al. How much demand for New HIV prevention technologies can we really expect? Results from a discrete choice experiment in South Africa. PLoS One. 2013;8(12):e83193.

37. Bridges JF, et al. Designing family-centered male circumcision services: a conjoint analysis approach. Patient. 2012;5(2):101-11.

38. Bridges JFP, et al. Engaging families in the choice of social marketing strategies for male circumcision services in Johannesburg, South Africa. Social Market Q. 2010;16(3):60-76.

39. Bridges JF, et al. Condom avoidance and determinants of demand for male circumcision in Johannesburg, South Africa. Health Policy Plan. 2011;26(4):298-306.

40. Dubov A, et al. Optimizing access to PrEP based on MSM preferences: results of a discrete choice experiment. AIDS Care. 2019;31(5):545-53.

41. Nwaozuru U, et al. Preferences for HIV testing services among young people in Nigeria. BMC Health Serv Res. 2019;19(1):1003. 
42. Kruk ME, et al. How can the health system retain women in HIV treatment for a lifetime? A discrete choice experiment in Ethiopia and Mozambique. PLoS One. 2016;11(8):e0160764.

43. Lancaster KE, et al. Preferences for pre-exposure prophylaxis service delivery among female sex workers in Malawi: a discrete choice experiment. AIDS Behav. 2020;24(5):1294-303.

44. Ostermann J, et al. Heterogeneous HIV testing preferences in an urban setting in Tanzania: results from a discrete choice experiment. PLoS One. 2014;9(3):e92100.

45. Ostermann J, et al. One size does not fit all: HIV testing preferences differ among high-risk groups in Northern Tanzania. AIDS Care. 2015;27(5):595-603.

46. Quaife M, et al. Divergent preferences for HIV prevention: a discrete choice experiment for multipurpose HIV prevention products in South Africa. Med Decis Making. 2018;38(1):120-33.

47. Zanolini A, et al. HIV self-testing in Lusaka Province, Zambia: acceptability, comprehension of testing instructions, and individual preferences for self-test kit distribution in a population-based sample of adolescents and adults. AIDS Res Hum Retrovirus. 2018;34(3):254-60.

48. Loutfy M, et al. Pregnancy planning preferences among people and couples affected by human immunodeficiency virus: piloting a discrete choice experiment. Int J STD AIDS. 2018;29(4):382-91.

49. Browne EN, et al. Efficacy is not everything: eliciting women's preferences for a vaginal HIV prevention product using a discrete-choice experiment. AIDS Behav. 2020;24(5):1443-51.

50. Maddala T, Phillips KA, Johnson FR. An experiment on simplifying conjoint analysis designs for measuring preferences. Health Econ. 2003;12(12):1035-47.

51. Minnis A, et al. Young women's stated preferences for biomedical HIV prevention: results of a discrete choice experiment in Kenya and South Africa. J Acquir Immune Defic Syndr. 2019;80(4):394-403.

52. Ong JJ, et al. Use of lotteries for the promotion of voluntary medical male circumcision service: a discrete-choice experiment among adult men in Tanzania. Med Decis Making. 2019;39(4):474-85.

53. Schaffer EM, et al. Promoting HIV testing by men: a discrete choice experiment to elicit preferences and predict uptake of community-based testing in Uganda. Appl Health Econ Health Policy. 2020;18(3):413-32.

54. Bristow CC, et al. Attributes of diagnostic tests to increase uptake of dual testing for syphilis and HIV in Port-au-Prince. Haiti. Int J STD AIDS. 2017;28(3):259-64.

55. Lee SJ, et al. Assessing willingness to test for HIV among men who have sex with men using conjoint analysis, evidence for uptake of the FDA-approved at-home HIV test. AIDS Care. 2013;25(12):1592-8.

56. Newman PA, et al. Willingness to participate in HIV vaccine trials: the impact of trial attributes. Prev Med. 2007;44(6):554-7.

57. Newman PA, et al. HIV vaccine acceptability among communities at risk: the impact of vaccine characteristics. Vaccine. 2006;24(12):2094-101.

58. Newman PA, et al. Preventive HIV vaccine acceptability and behavioral risk compensation among a random sample of highrisk adults in Los Angeles (LA VOICES). Health Serv Res. 2009;44(6):2167-79.

59. Newman PA, et al. Preventive HIV vaccine acceptability and behavioral risk compensation among high-risk men who have sex with men and transgenders in Thailand. Vaccine. 2010;28(4):958-64.

60. Weaver J, et al.,'Sisters, mothers, daughters and aunties': HIV vaccine acceptability among African, Caribbean and other Black women in Toronto. Can J Public Health. 2013;104(5):e413-7.
61. Galea JT, et al. Acceptability of pre-exposure prophylaxis as an HIV prevention strategy: barriers and facilitators to pre-exposure prophylaxis uptake among at-risk Peruvian populations. Int J STD AIDS. 2011;22(5):256-62.

62. Kinsler JJ, et al. Using conjoint analysis to measure the acceptability of rectal microbicides among men who have sex with men in four South American cities. AIDS Behav. 2012;16(6):1436-47.

63. Lee $\mathrm{S}$, et al. HIV vaccine acceptability among immigrant Thai residents in Los Angeles: a mixed-method approach. AIDS Care. 2008;20(10):1161-8.

64. Pines HA, et al. Oral and vaginal HIV pre-exposure prophylaxis product attribute preferences among female sex workers in the Mexico-US border region. Int J STD AIDS. 2019;30(1):45-55.

65. Shrestha R, et al. Measuring acceptability and preferences for implementation of pre-exposure prophylaxis (PrEP) using conjoint analysis: an application to primary HIV prevention among high risk drug users. AIDS Behav. 2018;22(4):1228-38.

66. Dubov A, et al. Strategies to implement pre-exposure prophylaxis with men who have sex with men in Ukraine. AIDS Behav. 2018;22(4):1100-11.

67. Primrose RJ, et al. Drivers of vaginal drug delivery system acceptability from internet-based conjoint analysis. PLoS One. 2016;11(3):e0150896.

68. Sharma A, et al. Acceptability and intended usage preferences for six HIV testing options among internet-using men who have sex with men. Springerplus. 2014;3:109.

69. Tanner AE, et al. Vaginal microbicide preferences among midwestern urban adolescent women. J Adolesc Health. 2008;43(4):349-56.

70. Eisingerich AB, et al. Attitudes and acceptance of oral and parenteral HIV preexposure prophylaxis among potential user groups: a multinational study. PLoS One. 2012;7(1):e28238.

71. Wheelock A, et al. Are Thai MSM willing to take PrEP for HIV prevention? An analysis of attitudes, preferences and acceptance. PLoS One. 2013;8(1):e54288.

72. Ravert RD, Zimet GD. College student invulnerability beliefs and HIV vaccine acceptability. Am J Health Behav. 2009;33(4):391-9.

73. Aliyu G, et al. HIV infection awareness and willingness to participate in future HIV vaccine trials across different risk groups in Abuja, Nigeria. AIDS Care. 2010;22(10):1277-84.

74. Fisman DN, et al. Willingness to pay to avoid sharps-related injuries: a study in injured health care workers. Am J Infect Control. 2002;30(5):283-7.

75. Flowers P, et al. Preparedness for use of the rapid result HIV self-test by gay men and other men who have sex with men (MSM): a mixed methods exploratory study among MSM and those involved in HIV prevention and care. HIV Med. 2017;18(4):245-55.

76. Halpern SD, et al. Who will enroll? Predicting participation in a phase II AIDS vaccine trial. J Acquir Immune Defic Syndr. 2001;27(3):281-8.

77. Izizag BB, et al. Factors associated with acceptability of HIV self-testing (HIVST) among university students in a peri-urban area of the Democratic Republic of Congo (DRC). Pan Afr Med J. 2018;31:248.

78. Morgan J, et al. Awareness of, interest in, and willingness to pay for HIV pre-exposure prophylaxis among Canadian gay, bisexual, and other men who have sex with men. Can J Public Health. 2018;109(5-6):791-9.

79. Oldenburg CE, et al. Antiretroviral pre-exposure prophylaxis preferences among men who have sex with men in Vietnam: results from a nationwide cross-sectional survey. Sex Health. 2016 Jul 22. https://doi.org/10.1071/sh15144. https://doi. org/10.1071/sh15144. [Epub ahead of print]. 
80. Robinson WT, Zarwell M, Gruber D. Preferences for home-based HIV testing among heterosexuals at increased risk for HIV/ AIDS: New Orleans, Louisiana, 2013. J Acquir Immune Defici Syndr. 2017;75(Suppl. 3):S352-6.

81. Sanders SA, et al. Women's willingness to experiment with condoms and lubricants: a study of women residing in a high HIV seroprevalence area. Int J STD AIDS. 2018;29(4):367-74.

82. Sharma A, Sullivan PS, Khosropour CM. Willingness to take a free home HIV test and associated factors among internet-using men who have sex with men. J Int Assoc Physicians AIDS Care (Chic). 2011;10(6):357-64.

83. Weinrib R, et al. End-users' product preference across three multipurpose prevention technology delivery forms: baseline results from young women in Kenya and South Africa. AIDS Behav. 2018;22(1):133-45.

84. Xun $\mathrm{H}$, et al. Factors associated with willingness to accept oral fluid HIV rapid testing among most-at-risk populations in China. PLoS ONE. 2013;8(11):e80594.

85. Zaveri T, et al. Firmness perception influences women's preferences for vaginal suppositories. Pharmaceutics. 2014;6(3):512-29.

86. Balán I, et al. Fingerprick versus oral swab: acceptability of blood-based testing increases if other STIs can be detected. AIDS Behav. 2017;21(2):501-4.

87. Castro JG, Jones DL, Weiss SM. STD patients' preferences for HIV prevention strategies. HIV/AIDS (Auckl). 2014;6:171-5.

88. Davide SH, et al. Patients' willingness to participate in rapid HIV testing: a pilot study in three New York City dental hygiene clinics. J Dent Hyg. 2017;91(6):41-8.

89. Durall PS, et al. Attitude toward rapid HIV testing in a dental school clinic. Spec Care Dentist. 2015;35(1):29-36.

90. Gilson R, Clutterbuck D, Chen Z. Demand for pre-exposure prophylaxis for HIV and the impact on clinical services: Scottish men who have sex with men perspectives. Int J STD AIDS. 2018;29(3):273-7.

91. Parsons JT, et al. Familiarity with and preferences for oral and long-acting injectable HIV pre-exposure Ppophylaxis (PrEP) in a national sample of gay and bisexual men in the U.S. AIDS Behav. 2016;20(7):1390-9.

92. Evans W, et al. Willingness to pay for condoms among men in sub-saharan Africa. Int J Environ Res Public Health. 2019;16(1):34.

93. Spielberg F, et al. Overcoming barriers to HIV testing: preferences for new strategies among clients of a needle exchange, a sexually transmitted disease clinic, and sex venues for men who have sex with men. J Acquir Immune Defic Syndr. 2003;32(3):318-27.

94. Whittington D, et al. Household demand for preventive HIV/ AIDS vaccines in Thailand: do husbands' and wives' preferences differ? Value Health. 2008;11(5):965-74.

95. Yuan L, et al. Factors associated with willingness to participate in free HIV test among general residents in Heilongjiang, Northeast China. BMC Infect Dis. 2012;12:256.

96. Uzochukwu B, et al. Voluntary counseling and testing (VCT) for HIV/AIDS: a study of the knowledge, awareness and willingness to pay for VCT among students in tertiary institutions in Enugu State Nigeria. Health Policy. 2011;99(3):277-84.

97. Conserve DF, et al. Sexual and social network correlates of willingness to self-test for HIV among ever-tested and never-tested men: implications for the Tanzania STEP Project. AIDS Care. 2019;31(2):169-76.

98. Carballo-Dieguez A, et al. Will gay and bisexually active men at high risk of infection use over-the-counter rapid HIV tests to screen sexual partners? J Sex Res. 2012;49(4):379-87.
99. Ostermann J, et al. Would you test for 5000 Shillings? HIV risk and willingness to accept HIV testing in Tanzania. Health Econ Rev. 2015;5(1):60.

100. Chiwaula LS, et al. The value of informal care in the context of option B plus in Malawi: a contingent valuation approach. BMC Health Serv Res. 2016;16:136.

101. Quaife M, et al. Preferences for ARV-based HIV prevention methods among men and women, adolescent girls and female sex workers in Gauteng Province, South Africa: a protocol for a discrete choice experiment. BMJ Open. 2016;6(6):e010682.

102. Forsythe $\mathrm{S}$, et al. Assessing the cost and willingness to pay for voluntary HIV counselling and testing in Kenya. Health Policy Plan. 2002;17(2):187-95.

103. Lee SJ, et al. Use of conjoint analysis to assess HIV vaccine acceptability: feasibility of an innovation in the assessment of consumer health-care preferences. Int J STD AIDS. 2012;23(4):235-41.

104. Whittington D, et al. Private demand for a HIV/AIDS vaccine: evidence from Guadalajara, Mexico. Vaccine. 2002;20(19-20):2585-91.

105. Newman P, et al. Acceptability and preferences for hypothetical rectal microbicides among a community sample of young men who have sex with men and transgender women in Thailand: a discrete choice experiment. AIDS Behav. 2016;20(11):2588-601.

106. Tang EC, et al. Using conjoint analysis to determine the impact of product and user characteristics on acceptability of rectal microbicides for HIV prevention among Peruvian men who have sex with men. Sex Transm Infect. 2016;92(3):200-5.

107. Verguet S, Holt BY, Szeri AJ. Increasing the effectiveness of vaginal microbicides: a biophysical framework to rethink behavioral acceptability. PLoS One. 2010;5(11):e15501.

108. Gonzalez JM. A guide to measuring and interpreting attribute importance. Patient. 2019;12(3):287-95.

109. Hollin IL, et al. Reporting formative qualitative research to support the development of quantitative preference study protocols and corresponding survey instruments: guidelines for authors and reviewers. Patient. 2020;13(1):121-36.

110. Beksinska M, et al. Offering choice in female condoms: the South African experience. Int J STD AIDS. 2019;30(10):985-90.

111. Pyra MN, et al. Global implementation of PrEP for HIV prevention: setting expectations for impact. J Int AIDS Soc. 2019;22(8):e25370.

112. Spinelli MA, Buchbinder SP. Pre-exposure prophylaxis persistence is a critical issue in PrEP implementation. Clin Infect Dis. 2020;71(3):583-5.

113. Mayer KH. Antiretroviral chemoprophylaxis: state of evidence and the research agenda. Clin Infect Dis. 2014;59(Suppl. 1):S47-51.

114. Montgomery ET, et al. End-user preference for and choice of four vaginally delivered HIV prevention methods among young women in South Africa and Zimbabwe: the Quatro Clinical Crossover Study. J Int AIDS Soc. 2019;22(5):e25283.

115. Griffin JB, et al. Vaginal ring acceptability and related preferences among women in low- and middle-income countries: a systematic review and narrative synthesis. PLoS One. 2019;14(11):e0224898.

116. Grimsrud A, et al. Shifting the narrative: from "the missing men" to "we are missing the men". J Int AIDS Soc. 2020;23(Suppl. 2):e25526

117. Beckham S, et al. Marked sex differences in all-cause mortality on antiretroviral therapy in low- and middle-income countries: a systematic review and meta-analysis. J Int AIDS Soc. 2016;19:22106.

118. NIH. Experimental HIV vaccine regimen ineffective in preventing HIV. Bethesda (MD): National Institutes of Health; 2020. 
119. Baral SD, et al. The epidemiology of HIV among men who have sex with men in countries with generalized HIV epidemics. Curr Opin HIV AIDS. 2014;9(2):156-67.

120. Baral S, et al. Burden of HIV among female sex workers in lowincome and middle-income countries: a systematic review and meta-analysis. Lancet Infect Dis. 2012;12(7):538-49.

121. Baral S, et al. Worldwide burden of HIV in transgender women: a systematic review and meta-analysis. Lancet Infect Dis. 2013;13:214-22.

122. Golub SA, et al. High rates of PrEP eligibility but low rates of PrEP access among a national sample of transmasculine individuals. J Acquir Immune Defic Syndr. 2019;82(1):e1-7.

123. Reisner SL, et al. High risk and low uptake of pre-exposure prophylaxis to prevent HIV acquisition in a national online sample of transgender men who have sex with men in the United States. J Int AIDS Soc. 2019;22(9):e25391.
124. PEPFAR. PEPFAR strategy for accelerating HIVAIDS epidemic control (2017-2020). Washington, DC: President's Emergency Fund for AIDS Relief (PEPFAR); 2017.

125. FDA. Patient preference information: voluntary submission, review in premarket approval applications, humanitarian device exemption applications, and de novo requests, and inclusion in decision summaries and device labeling. C.f.D.a.R.H. Department of Health and Human Services Food and Drug Administration, Center for Bioloigcs Evaluation and Research, editor. Washington, DC: Center for Devices and Radiological Health; 2016.

126. Medical Device Innovation Consortium. Patient centered benefit risk project report: a framework for incorporating information on patient preferences regarding benefit and risk into regulatory assessments of new medical technology. Arlington (VA): Medical Device Innovation Consortium; 2015. 